\title{
AN AVERAGING PRINCIPLE FOR FAST DEGREES OF FREEDOM EXHIBITING LONG-TERM CORRELATIONS*
}

\author{
CHRISTOF SCHÜTTE ${ }^{\dagger}$, JESSIKA WALTER ${ }^{\dagger}$, \\ CARSTEN HARTMANN ${ }^{\dagger}$, AND WILHELM HUISINGA ${ }^{\dagger}$
}

\begin{abstract}
This article is concerned with the averaging principle and its extensions for stochastic dynamical systems with fast and slow degrees of freedom. It is demonstrated how the "conventional" averaging principle results from asymptotic multiscale analysis, how one can construct an indicator for its (in-)appropriateness, and how, if inappropriate, it may be extended into an improved approximation. The conventional scheme contains averages over the entire accessible state space of the fast degrees of freedom and may thus fail if these fast degrees of freedom exhibit long-term (auto-)correlations. In contrast, the improved scheme combines several conditional averages with a Markov jump process that is designed to represent the flipping process between the conditional averages and thus incorporates the important long-term correlations. All important steps of the derivation are illustrated by numerical experiments. Application to problems from molecular dynamics is discussed.
\end{abstract}

Key words. diffusion process, multiscale asymptotics, deviations from averaging, conditional averaging, transfer operator approach, metastability, dominant spectrum, Fixman potential, conformational free energy landscape, Fokker-Planck generator, long-term correlations

AMS subject classifications. 60J60, 65U05, 41A60

DOI. $10.1137 / 030600308$

1. Introduction. Most realistically complex systems from fields like biomolecular dynamics, climate research, or materials science exhibit a rich hierarchy of temporal scales. One of the main strategies of dealing with the enormous complexity of the corresponding models is dimension reduction: describe the effective dynamical behavior in terms of some slow (or essential) degrees of freedom (DOF) of the system while the collective effect of the majority of fast (or inessential) DOF is represented implicitly.

Many different mathematical techniques are available, e.g., averaging for deterministic [2, 23, 48] and stochastic dynamical systems [22, 23, 31, 32, 33] (often in combination with large deviation theory), stochastic modeling [39, 40, 43, 56], adiabatic theories $[5,8,26,52]$, or homogenization in time $[6,7,8]$. These techniques have mathematically been considered in a variety of articles. Another branch of the literature is concerned with applications to real-world complex systems. In this direction the present article focuses on molecular dynamics applications; from this perspective one should at least mention the following three fields: First, we discuss heat bath effects, i.e., the idea of describing the collective effect of many (simple) fast DOF on some slow modes in terms of simple stochastic processes; this idea has influenced a lot of investigations in the direction of stochastic modeling; the most recent ones $[11,12,36]$ originate from the famous Zwanzig-Mori-Kac approach $[43,56]$. Second,

\footnotetext{
* Received by the editors May 30, 2003; accepted for publication (in revised form) January 24, 2004; published electronically June 15, 2004.

http://www.siam.org/journals/mms/2-3/60030.html

${ }^{\dagger}$ Institut für Mathematik II, Freie Universität Berlin, Arnimallee 2-6, 14195 Berlin, Germany (schuette@math.fu-berlin.de, walter@math.fu-berlin.de, chartman@math.fu-berlin.de, huisinga@ math.fu-berlin.de). The second author's research was supported by the DFG priority program "Analysis, Modeling and Simulation of Multiscale Problems" (SPP 1095). The research of the third and fourth authors was supported by the DFG Research Center Berlin "Mathematics for Key Technologies" (FZT 86).
} 
we analyze strong constraining potentials $[45,46,54]$ with their strong relations to both homogenization in time and averaging. And third, we examine the problem of resonances between the fast DOF, which is of immense importance for the discussion of strong constraining potentials $[6,48,54]$ as well as in the context of adiabatic theories for quantum dynamics $[18,27,38,52,55]$.

Focus. Despite this wealth of contributions, surprisingly few articles deal with the question of when these approximations may be inappropriate to reproduce the effective dynamics of the original system, and little information is available on the problem of how to eventually improve them. The present article will focus on exactly these two questions. The elimination scheme that we will consider is an averaging procedure with close relations to stochastic modeling. Our aim is to design robust indicators for its validity and to construct an extended version in the case in which the original technique is found to fail.

The averaging principle. Averaging results are known for deterministic dynamical systems, e.g., [2], as well as for stochastic ones, e.g., [23]. For example, they apply to systems with slow DOF that vary on time scale $\mathcal{O}(1)$ and fast DOF on time scale $\mathcal{O}(\epsilon)$. Averaging theorems then describe the effective motion of the slow DOF in the limit $\epsilon \rightarrow 0$. Their statement is based on a decoupling of fast and slow motion:

For any given position of the slow DOF, there is an accessible part of the state space that the fast DOF completely explore before the position of the slow ones changes effectively. The fast variables are eliminated from the original equation of motion by averaging according to the probability distribution corresponding to the exploration of the accessible state space. The effective motion of the slow variables is governed by an averaged equation of motion.

For illustration let us consider the following SDE:

$$
\begin{aligned}
\dot{x}^{\epsilon} & =-D_{x} V(x, y)+\sigma \dot{W}_{1}, \\
\epsilon \dot{y}^{\epsilon} & =-D_{y} V(x, y)+\sqrt{\epsilon} \sigma \dot{W}_{2},
\end{aligned}
$$

with $\epsilon>0, D_{x} V, D_{y} V$ denoting the derivatives of the potential $V=V(x, y)$ w.r.t. $x \in \mathbf{R}^{m}, y \in \mathbf{R}^{n}$, respectively, and $W_{j}(j=1,2)$ standard Brownian motion. For $\epsilon \ll 1$, this system consists of a fast variable, $y$, and a slow one, $x$. It is known that the SDE (1.1) and (1.2) has an invariant measure $\mu(\mathrm{d} x \mathrm{~d} y)=\mu(x, y) \mathrm{d} x \mathrm{~d} y$ with smooth density:

$$
\mu(x, y)=\frac{1}{Z} \exp (-\beta V(x, y)), \quad Z=\int \exp (-\beta V(x, y)) \mathrm{d} x \mathrm{~d} y,
$$

which depends on the inverse temperature $\beta=2 / \sigma^{2}$. In the following we assume that this is the unique invariant measure (sufficient conditions will be given below). In this case (and under suitable conditions for $V$; cf. [23]) averaging completely characterizes the limit $x^{0}$ of the slow dynamics $x^{\epsilon}$ for $\epsilon \rightarrow 0$ obeying an averaged SDE,

$$
\dot{x}^{0}=-D_{x} \bar{V}\left(x^{0}\right)+\sigma \dot{W}_{1} .
$$

Here, the averaged force $D_{x} \bar{V}(x)$ on $x$ is given by

$$
\begin{aligned}
D_{x} \bar{V}(x) & =\frac{1}{Z_{x}} \int D_{x} V(x, y) \exp (-\beta V(x, y)) \mathrm{d} y, \\
Z_{x} & =\int \exp (-\beta V(x, y)) \mathrm{d} y
\end{aligned}
$$


which is the gradient of the averaged potential,

$$
\bar{V}(x)=-\frac{1}{\beta} \log \int \exp (-\beta V(x, y)) \mathrm{d} y .
$$

In the case of mechanical systems the averaged potential is strongly related to the socalled potential of mean force or conformational free energy landscape [3, 10, 34, 53], which are both well known from biomolecular applications.

When averaging fails. Our decisive question is, Under which circumstances may the averaging principle fail, even for significantly small values of $\epsilon$ ? Since the averaging principle is based on the fact that the fast DOF explore the accessible state space before any effective change in the slow DOF, our question can be reformulated as,

What can prevent the fast DOF for given small $\epsilon>0$ from sampling the accessible state space entirely before the slow ones effectively change?

This obviously happens if there is some subset of the accessible state space from which the fast motion will most probably exit only on some scale of order $\mathcal{O}(1)$. This phenomenon is related to metastability in the dynamics, i.e., to the existence of metastable subsets in the state space $[14,51,30,50]$. Consequently, the main part of the article is concerned with the effect of metastable subsets in the accessible state space of the fast DOF on the limit motion of the slow ones.

Averaging, metastability, and transfer operators. Several recent articles address the relation of metastability and dominant eigenmodes of the transfer operator associated with the dynamical system $[14,29,30,50,51]$. We will exploit these results in order to explain possible deviations of the full motion from the averaged motion. This explanation will also allow us to construct an indicator for these deviations. Furthermore, it will lead towards the derivation of a principle of conditional averaging that, under certain circumstances, yields an appropriate reduced model in cases where the "usual" averaging principle fails. Moreover, the resulting numerical scheme can be handled almost as easily as the usual averaging scheme.

Conditional averaging. In the case considered above, the conditional averaging scheme has the following form: Suppose that for fixed slow DOF $x$ the accessible state space of the fast variable $y$ can be decomposed into $m(x)$ metastable subsets $B_{i}(x)$. Assume, moreover, that, for the sake of simplicity, the sets $B_{i}(x)$ if appropriately ordered depend sufficiently regularly on $x$. Moreover, let $I(x)$ denote a Markov jump process with state space $\{1, \ldots, m(x)\}$ whose transition rates are given by the expected transition rates between the $m(x)$ metastable subsets. Then the conditionally averaged limit dynamics has the form

$$
\begin{aligned}
\dot{x}^{0} & =-D_{x} \bar{V}^{\left(I\left(x^{0}\right)\right)}\left(x^{0}\right)+\sigma \dot{W}_{1}, \\
\bar{V}^{(i)}(x) & =-\frac{1}{\beta} \log \int_{B_{i}(x)} \exp (-\beta V(x, y)) \mathrm{d} y .
\end{aligned}
$$

That is, the limit dynamics jumps between averaged potentials, each of them being averaged over one of the metastable subsets, and the rates of the jumps reproduce the transition rates of the original system. We will give a derivation of this limit dynamics and present numerical experiments that indicate its reliability.

The phrase conditional averaging is used in several significantly different contexts, for example in spatial averages in turbulence modeling or averages w.r.t. stationary conditional probability distributions in biomolecular structure description [47]. The 
present authors are not aware of any notion of conditional averaging that is comparable to the concept to be presented herein, i.e., that is tailored to the incorporation of temporal fast scale effects and can be derived by means of linear projection techniques.

The need for conditional averaging in molecular dynamics. Molecular dynamics is concerned with classical or quantum Hamiltonian equations of motion, often purely deterministic but also stochastically perturbed (so-called Langevin dynamics; see $[1,49]$, for example). The basic constituent of all descriptions is the potential energy that models all covalent, ionic, or associative interactions of atoms in the molecule. The inherent temporal multiscale structure of the associated Hamiltonian system spans over several orders of magnitude, from the fastest time scales on the femtosecond scale $\left(10^{-15} \mathrm{~s}\right)$ up to the slowest typically beyond the microseconds $\left(10^{-6} \mathrm{~s}\right)$. The fastest motions are certain bond length, bond angle, or torsion angle oscillations that are related (a) to strong constraining parts of the potential and/or (b) to small parameters, in particular small effective masses. Fast torsion angle modes are of particular interest for this article: While bond lengths and bond angles typically have only one equilibrium position, torsion angle dynamics is determined by multiwell potentials. These multiwell structures can contain significantly large energy barriers such that the corresponding (fast) torsion angle mode may exhibit metastability. The elimination of fast torsion angle modes will thus make conditional averaging necessary.

Outline. We will proceed as follows: First, we introduce the stochastic dynamical systems under consideration. Then we will briefly review the transfer operator approach to metastability. The main part of the article exploits asymptotic multiscale techniques to state the averaging principle, to indicate when averaging possibly fails, and to derive the conditional averaging scheme. Finally, we will illustrate all these steps by suitable, numerical experiments and discuss the possible applicability of the concept to realistic molecular dynamics problems and related fields.

2. The system under consideration. In the following we will focus on diffusion processes with fast and slow scales; i.e., we will return to the SDE (1.1) and (1.2) from the introduction. Under reasonable conditions it has a unique and smooth invariant density, a property that excludes many nasty questions we are not interested in at this time. The following sections will be concerned with the approximation of the full dynamics given by the SDE (1.1) and (1.2) on finite time scales of order $\mathcal{O}(1)$; i.e., we will not consider $\mathcal{O}(1 / \epsilon)$ or even longer periods. Furthermore, we will ignore the case of fast scales originating from strong constraining potentials with strong parts increasing with $\epsilon$, i.e., of the form $V(x, y)=U(x)+\mathcal{V}(x, y) / \epsilon^{2}$.

Consider the Hilbert space

$$
L^{2}(\mu)=\left\{g=g(x, y): \quad \int|g(x, y)|^{2} \mu(x, y) \mathrm{d} x \mathrm{~d} y<\infty\right\}
$$

with the usual scalar product

$$
\langle g, f\rangle_{\mu}=\int f(x, y) \overline{g(x, y)} \mu(x, y) \mathrm{d} x \mathrm{~d} y
$$

where $\bar{z}$ denotes the complex conjugate of $z$. We want to describe the evolution of the probability density $\rho^{\epsilon}$ under the dynamics given by (1.1) and (1.2). For reasons that become clear later we are interested in probability densities weighted w.r.t. $\mu$; i.e., $\rho^{\epsilon}(x, y, t) \mu(x, y)$ is the usually considered physical probability density function to find the system in $(x, y)$ at time $t$ after having started with the probability density $\rho^{\epsilon}(x, y, t=0) \mu(x, y)$ at $t=0$. 
Consequently, we consider the Fokker-Planck equation corresponding to (1.1) and (1.2) in the $\mu$-weighted space, which then reads

$$
\begin{aligned}
\partial_{t} \rho^{\epsilon} & =\mathcal{L}_{\epsilon} \rho^{\epsilon}, \\
\mathcal{L}_{\epsilon} & =\frac{1}{\epsilon} \mathcal{L}_{x}+\mathcal{L}_{y}, \\
\mathcal{L}_{x} & =\frac{\sigma^{2}}{2} \Delta_{y}-D_{y} V(x, \cdot) \cdot D_{y}, \\
\mathcal{L}_{y} & =\frac{\sigma^{2}}{2} \Delta_{x}-D_{x} V(\cdot, y) \cdot D_{x} .
\end{aligned}
$$

The indices of the operators $\mathcal{L}_{x}$ and $\mathcal{L}_{y}$ indicate the coordinate that can be considered fixed for the respective operation; e.g., $\mathcal{L}_{x}$ can be considered as a differential operator acting on $y$ only, where $x$ is meant to be fixed. The usefulness of this notation will become obvious subsequently.

In section 4.2 we study the case where the initial condition $\rho^{\epsilon}(x, y, 0)=f(x)$ depends on $x$ only; i.e., only the slow variable is observed. For preparation of this discussion we define the projection operator $\Pi$ :

$$
\begin{aligned}
(\Pi g)(x) & =\int g(x, y) \mu_{x}(y) \mathrm{d} y, \\
\mu_{x}(y) & =\frac{1}{Z_{x}} \exp (-\beta V(x, y)), \quad \beta=\frac{2}{\sigma^{2}}, \\
Z_{x} & =\int \exp (-\beta V(x, y)) \mathrm{d} y .
\end{aligned}
$$

It is obvious that $\Pi$ projects on functions which do not depend on $y$. That is, we can express the restriction on the initial condition by means of

$$
\left(\Pi \rho^{\epsilon}\right)(\cdot, t=0)=\rho^{\epsilon}(\cdot, \cdot, t=0) .
$$

It is likewise obvious that $\mathcal{L}_{x} \Pi=0$ since $\mathcal{L}_{x}$ does act only on $y$. Due to the properties of $\mathcal{L}_{x}$, we furthermore have (for all functions within the domain of definition)

$$
\Pi \mathcal{L}_{x}=0=\mathcal{L}_{x} \Pi .
$$

The aim is to obtain a valid representation of the effective dynamics of (1.1) and (1.2) in the slow variable $x$ only. Whatever this representation may look like, the knowledge of the invariant measure $\mu$ of the full dynamics already tells us the form of the invariant measure of the reduced dynamics:

$$
\bar{\mu}(x)=\frac{1}{Z} \int \exp (-\beta V(x, y)) \mathrm{d} y .
$$

3. Dominant spectra and metastability. There are several recent articles on the relation between metastability and dominant eigenmodes of the transfer operator related to the considered dynamical system [14, 29, 30, 50, 51]. Within these approaches, metastability is a setwise notion conceptually defined in the following way: Some dynamical system is said to exhibit metastability or to have a metastable decomposition if its state space can be decomposed into a finite (hopefully small) number of disjoint sets such that transitions between these sets are extremly rare [14, 51]. There are basically two different concepts to quantify the "rareness of transitions": (a) via 
transition probabilities w.r.t. an ensemble of systems and measured as the fraction of systems that exit the set during some fixed finite time interval $[50,51]$ and (b) in the case of a stochastic process via the expected exit probability [9] or via the decay rate of the distribution of exit times [30]. However, both concepts (a) and (b) are related to the dominant eigenvectors of the transfer operator. Accordingly, the basic insight of the transfer operator approach to metastability is as follows [51]:

Identification of metastable decompositions. Metastable decompositions can be detected via the discrete eigenvalues of the transfer operator $P_{\epsilon}^{t}$ close to its maximal eigenvalue $\lambda=1$; they can be identified by exploiting structural properties of the corresponding eigenfunctions. In doing so, the number of sets in the metastable decomposition is equal to the number of eigenvalues close to 1 , including $\lambda=1$ and counting multiplicity.

For the diffusion processes $X^{\epsilon}=\left(x^{\epsilon}, y^{\epsilon}\right)$ the transfer operator $P_{\epsilon}^{t}$ is generated by the differential operator $\mathcal{L}_{\epsilon}$ from (2.2), i.e.,

$$
P_{\epsilon}^{t}=\exp \left(t \mathcal{L}_{\epsilon}\right) \quad \text { in } L^{2}(\mu)
$$

with a suitable extension of $\mathcal{L}_{\epsilon}$ to $L^{2}(\mu)$ (cf. [30]).

3.1. Dominant spectrum of the generator in $L^{2}(\mu)$. Since $\mathcal{L}_{\epsilon}$ is self-adjoint in $L^{2}(\mu)$ its spectrum is real-valued. Moreover, it is nonpositive with largest eigenvalue $\lambda_{0}=0$ such that eigenvalues of $P_{\epsilon}^{t}$ close to its largest eigenvalue 1 correspond to those of $\mathcal{L}_{\epsilon}$ close to (but smaller than) $\lambda_{0}=0$, while the eigenfunctions are the same in both cases. Consequently, we can extract metastable decompositions from the dominant eigenfunctions of the generator $\mathcal{L}_{\epsilon}$. The existence of a metastable decomposition into $M$ sets is related in the following manner to the spectrum of $\mathcal{L}_{\epsilon}$.

Assumption 1. There is some positive radius $R \ll 1$ such that the intersection of the $L^{2}(\mu)$-spectrum of $\mathcal{L}_{\epsilon}$ with the interval $[-R, 0]$ is discrete and contains $M$ isolated eigenvalue $0=\lambda_{0}>\lambda_{1} \geq \cdots \geq \lambda_{M-1}$, where the eigenvalues are repeated according to their multiplicity.

Rigorous statements on the relation between the dominant spectrum in this sense and the existence of metastable decomposition can be found in [9, 16, 29, 30, 51]. Here, we mention only that whenever the process $X^{\epsilon}$ is geometrically ergodic then the largest eigenvalue $\lambda_{0}$ is an isolated, simple eigenvalue [42]; in [41] sufficient conditions for geometrical ergodicity are given in terms of smoothness and growth of the potential. The eigenfunction corresponding to the largest eigenvalue $\lambda_{0}=0$ of $\mathcal{L}_{\epsilon}$ is simply given by the constant function $1 \equiv \mathbf{1}(x, y)$ :

$$
\mathcal{L}_{\epsilon} \mathbf{1}=0
$$

Let us now assume that the spectrum of $\mathcal{L}_{\epsilon}$ has the form described above, and suppose that $M=2$. Let us denote the eigenfunctions associated with the isolated eigenvalues $\lambda_{0}=0$ and $\lambda_{1}<0$ by $u_{0}$ and $u_{1}$, respectively. Thus we have a metastable decomposition of the state space $\mathbf{X}$ into two disjoint sets, $B$ and its complement $B^{c}=\mathbf{X} \backslash B$, which are defined by the zeros of the second eigenfunction in the following manner:

$$
B=\left\{(x, y): u_{1}(x, y)<0\right\} \text { and } B^{c}=\left\{(x, y): u_{1}(x, y) \geq 0\right\} .
$$

Furthermore, the results of [30] tell us that the second eigenfunction $u_{1}$ can be approximated by

$$
u_{B}=\sqrt{\frac{\mu\left(B^{c}\right)}{\mu(B)}} \mathbf{1}_{B}-\sqrt{\frac{\mu(B)}{\mu\left(B^{c}\right)}} \mathbf{1}_{B^{c}}
$$


where $\mathbf{1}_{B}$ denotes the indicator function of the set $B \subset \mathbf{X}$. In fact, the results of $[29,30]$ show that the deviation $\left\|u_{1}-u_{B}\right\|_{\mu}$ decays exponentially with decreasing noise amplitude $\sigma$. The function $u_{B}$ is constant on either of the two sets $B$ and $B^{c}$, is normalized to $\left\|u_{B}\right\|_{2}=1$, and satisfies $\left\langle\mathbf{1}_{\mathbf{X}}, u_{B}\right\rangle_{\mu}=0$.

3.2. Freezing the slow DOF. The operators $\Pi$ and $\mathcal{L}_{x}$ can also be considered as families of operators acting on the variable $y$ for fixed $x$. That is, we are interested in the action on the fiber

$$
\Phi(x)=\left\{(x, y): \quad y \in \mathbf{R}^{n}\right\} .
$$

For functions depending on $x$ and $y$, say $g=g(x, y)$, we then take $x$ as a fixed parameter and introduce the family of Hilbert spaces

$$
L^{2}\left(\mu_{x}\right)=\left\{g=g(y): \int|g(y)|^{2} \mu_{x}(y) \mathrm{d} y<\infty\right\} \quad \text { on each } \Phi(x),
$$

with scalar products

$$
\langle f, g\rangle_{\mu_{x}}=\int f(y) \bar{g}(y) \mu_{x}(y) \mathrm{d} y .
$$

We know that $\mathcal{L}_{x}$ is self-adjoint on $L^{2}\left(\mu_{x}\right)$. Moreover, we observe that $\mathbf{1}(x, \cdot)$ is an eigenfunction of $\mathcal{L}_{x}$ for the eigenvalue $\lambda=0$. Additionally, if we assume that the Markov process given by (1.2) for fixed $x$ is geometrically ergodic, then $\mathbf{1}(x, \cdot)$ is the unique eigenfunction of $\mathcal{L}_{x}$ for the largest eigenvalue $\lambda=0$ in $L^{2}\left(\mu_{x}\right)$. Moreover, since we can rewrite $\Pi$ by

$$
\Pi g(x)=\langle\mathbf{1}, g\rangle_{\mu_{x}}
$$

and observe that $\Pi$, considered in $L^{2}\left(\mu_{x}\right)$, is the orthogonal projection onto the eigenspace of the lowest eigenvalue $\lambda=0$; i.e., $\Pi$ projects onto the kernel of $\mathcal{L}_{x}$.

In addition, geometric ergodicity guarantees that the eigenvalue $\lambda_{0}=0$ is known to be isolated in the $L^{2}\left(\mu_{x}\right)$-spectrum. Thus, the spectrum of $\mathcal{L}_{x}$ restricted to

$$
L_{0}^{2}\left(\mu_{x}\right)=\left\{g \in L^{2}\left(\mu_{x}\right): \Pi g=0\right\}
$$

is bounded away from zero; i.e., there is some $\delta_{x}>0$ such that

$$
\sup \sigma\left(\mathcal{L}_{x} \uparrow_{L_{0}^{2}\left(\mu_{x}\right)}\right)=-\delta_{x}<0
$$

As a consequence, the inverse exists and satisfies

$$
\|\left(\mathcal{L}_{x}\left\lceil_{L_{0}^{2}\left(\mu_{x}\right)}\right)^{-1} \|_{\mu_{x}} \leq \mid \sup \sigma\left(\left.\mathcal{L}_{x}\left\lceil_{L_{0}^{2}\left(\mu_{x}\right)}\right)\right|^{-1}=\delta_{x}^{-1} .\right.\right.
$$

3.3. Dominant spectrum via potential energy landscape $\boldsymbol{V}$. In the Wentzell-Freidlin setting of large deviation theory [23], relations between the dominant spectrum of the generator $\mathcal{L}$ or $\mathcal{L}_{x}$, respectively, and the potential energy landscape are studied. It can be shown that in the limit for vanishing noise amplitude $\sigma$, the dominant eigenvalues are related to the local minima of the potential energy landscape. More precisely, the eigenvalues correspond to the inverse mean exit time from small sets around each minimum [9]; information about corresponding exit rates also is available [30]. The exits from each local minimum take place close to the lowest 
saddle point that allows passing from one minimum to another. The corresponding exit times are exponentially small in terms of the corresponding potential barriers that the process has to pass. Thus, the eigenvalues nearest to zero are related to transitions between the most significant wells in the potential energy landscape. In some of the following steps this would allow us to exploit explicit asymptotic formulae for the dominant eigenvalues of $\mathcal{L}$ or $\mathcal{L}_{x}$, respectively. However, we will not pursue this direction since we are aiming at developing a theory that allows for generalization to dynamical systems for which the relation between metastability, dominant spectrum, and potential energy landscapes is not as obvious as for diffusion processes.

\section{Multiscale asymptotics.}

4.1. Multiscale asymptotics of eigenvalues and eigenvectors. We are interested in the eigenvalues and eigenvectors of the full generator $\mathcal{L}_{\epsilon}$. We therefore assume that $\lambda^{\epsilon}$ is some isolated eigenvalue of $\mathcal{L}_{\epsilon}$ with multiplicity one and corresponding eigenvector $f^{\epsilon}$. We make the following ansatz:

$$
\begin{aligned}
& f^{\epsilon}=f_{0}+\epsilon f_{1}+\epsilon^{2} f_{2}+\cdots, \\
& \lambda^{\epsilon}=\lambda_{0}+\epsilon \lambda_{1}+\cdots .
\end{aligned}
$$

Inserting this into the eigenvalue equation, we get via a comparison of powers of $\epsilon$

$$
\begin{aligned}
\epsilon^{-1}: & \mathcal{L}_{x} f_{0}=0 \\
\epsilon^{0}: & \mathcal{L}_{x} f_{1}+\mathcal{L}_{y} f_{0}=\lambda_{0} f_{0}, \\
\epsilon^{1}: & \mathcal{L}_{x} f_{2}+\mathcal{L}_{y} f_{1}=\lambda_{1} f_{0}+\lambda_{0} f_{1} .
\end{aligned}
$$

Step 1. (4.1) immediately yields that $f_{0}$ does not depend on $y$, i.e,

$$
\Pi f_{0}=f_{0} .
$$

Thus the eigenvectors of $\mathcal{L}_{\epsilon}$ are almost constant w.r.t. $y$ if $\epsilon$ is sufficiently small.

Step 2. Let $\Pi$ act on (4.2) and use (2.4). This time we have

$$
\lambda_{0} f_{0}=\Pi \mathcal{L}_{y} f_{0}=\Pi \mathcal{L}_{y} \Pi f_{0} .
$$

Let us abbreviate

$$
\overline{\mathcal{L}}=\Pi \mathcal{L}_{y} \Pi .
$$

Since $\mathcal{L}_{y}$ contains derivatives w.r.t. $x$ only, then we immediately see that

$$
\overline{\mathcal{L}}=\frac{\sigma^{2}}{2} \Delta_{x}-\left(\Pi D_{x} V\right) \cdot D_{x}, \quad \text { acting on } L^{2}(\bar{\mu}) .
$$

One easily computes that $\Pi D_{x} V$ can again be expressed as the gradient of the averaged potential, i.e., $\Pi D_{x} V=D_{x} \bar{V}$, with

$$
\bar{V}(x)=-\frac{1}{\beta} \log Z_{x},
$$

and therefore

$$
\overline{\mathcal{L}}=\frac{\sigma^{2}}{2} \Delta_{x}-D_{x} \bar{V} \cdot D_{x} \quad \text { on } L^{2}(\bar{\mu}) .
$$


Thus up to a deviation of order $\mathcal{O}(\epsilon)$ the isolated eigenvalues and associated eigenvectors of $\mathcal{L}_{\epsilon}$ are given by the corresponding ones of $\overline{\mathcal{L}}$, which again is a Fokker-Planck generator with averaged potential $\bar{V}$.

Obviously, the averaged potential $\bar{V}$ is the free energy landscape that has already been introduced. Next, we will see that the multiscale asymptotics if used for the dynamics itself results in averaging in lowest order and in second-lowest order tells us when averaging needs to be corrected.

4.2. Multiscale asymptotics and averaging. We now make the following ansatz for the solution of the Fokker-Planck equation (2.1) with the initial conditions described above:

$$
\rho^{\epsilon}=\rho_{0}+\epsilon \rho_{1}+\epsilon^{2} \rho_{2}+\cdots .
$$

This ansatz is inserted into the Fokker-Planck equation, and then, by comparison of coefficients of different powers of $\epsilon$, we get

$$
\begin{aligned}
\epsilon^{-1}: & \mathcal{L}_{x} \rho_{0}=0, \\
\epsilon^{0}: & \mathcal{L}_{x} \rho_{1}+\mathcal{L}_{y} \rho_{0}=\partial_{t} \rho_{0}, \\
\epsilon^{1}: & \mathcal{L}_{x} \rho_{2}+\mathcal{L}_{y} \rho_{1}=\partial_{t} \rho_{1} .
\end{aligned}
$$

Step 1. (4.6) immediately yields that $\rho_{0}$ does not depend on $y$, i.e,

$$
\Pi \rho_{0}=\rho_{0} .
$$

Step 2. Let $\Pi$ act on (4.7) and use (2.4). This time

$$
\partial_{t} \rho_{0}=\Pi \mathcal{L}_{y} \rho_{0}=\Pi \mathcal{L}_{y} \Pi \rho_{0} .
$$

Thus $\rho_{0}$ is determined by a Fokker-Planck equation with averaged potential, and its solution gives us $\rho^{\epsilon}$ up to error $\mathcal{O}(\epsilon)$. The associated SDE

$$
\dot{x}^{0}=-D_{x} \bar{V}\left(x^{0}\right)+\sigma \dot{W}_{1}
$$

thus describes the limit dynamics of (1.1) and (1.2) in the sense that its solution satisfies $x^{\epsilon} \rightarrow x^{0}$ as $\epsilon \rightarrow 0$ either pathwise [23] or in the distributional sense [37, 39]. Obviously, the invariant measure of the averaged dynamics (4.9) is given by $\bar{\mu}$ as given in (2.5). Obviously, we additionally can represent $\bar{\mu}$ via

$$
\bar{\mu}(x)=\frac{1}{Z} \exp (-\beta \bar{V}(x)) .
$$

Measuring deviations from averaged dynamics. Now, let $Q=1-\Pi$ act on (4.7). Since $Q \rho_{0}=0$ we get

$$
Q \mathcal{L}_{x} \rho_{1}+Q \mathcal{L}_{y} \rho_{0}=0 .
$$

Furthermore, (2.4) allows us to rewrite the parts of this equation:

$$
Q \mathcal{L}_{x} \rho_{1}=\mathcal{L}_{x} \rho_{1}=-Q \mathcal{L}_{y} \rho_{0}=\overline{\mathcal{L}} \rho_{0}-\mathcal{L}_{y} \rho_{0} .
$$

Thus, we get an equation for the first order deviation $\rho_{1}$ that depends on the difference between the averaged potential and the full one:

$$
\mathcal{L}_{x} \rho_{1}=\overline{\mathcal{L}} \rho_{0}-\mathcal{L}_{y} \rho_{0}=D_{x}(V-\bar{V}) \cdot D_{x} \rho_{0} .
$$


If we decompose $\rho_{1}$ into the part that is constant in the $y$-direction, $\Pi \rho_{1}$, and the part that is not, $\rho_{1}-\Pi \rho_{1}$, we can get an explicit expression for the latter part from the last equation: Since

$$
\Pi\left(\rho_{1}-\Pi \rho_{1}\right)=0
$$

the last formula can also be expressed as

$$
\mathcal{L}_{x}\left(\rho_{1}-\Pi \rho_{1}\right)=\left(\overline{\mathcal{L}}-\mathcal{L}_{y}\right) \rho_{0},
$$

which together with the estimate (3.3) yields a bound on the correction $\rho_{1}-\Pi \rho_{1}$ for every $x$ (at least for those for which the above assumption of geometrical ergodicity holds):

$$
\left\|\rho_{1}-\Pi \rho_{1}\right\|_{\mu_{x}} \leq \|\left(\mathcal{L}_{x}\left\lceil_{L_{0}^{2}\left(\mu_{x}\right)}\right)^{-1}\left\|_{\mu_{x}}\right\|\left(\overline{\mathcal{L}}-\mathcal{L}_{y}\right) \rho_{0}\left\|_{\mu_{x}}=\delta_{x}^{-1}\right\|\left(\overline{\mathcal{L}}-\mathcal{L}_{y}\right) \rho_{0} \|_{\mu_{x}},\right.
$$

since $\left(\overline{\mathcal{L}}-\mathcal{L}_{y}\right) \rho_{0} \in L_{0}^{2}\left(\mu_{x}\right)$. Summarizing we now know the following:

The smaller the spectral gap $\delta_{x}$ of $\mathcal{L}_{x}$ w.r.t. its lowest eigenvalue

$\lambda=0$ in $L^{2}\left(\mu_{x}\right)$, the larger $\left\|\rho_{1}-\Pi \rho_{1}\right\|_{\mu_{x}}$ may become.

Conclusively, if the inequality

$$
\frac{1}{\delta_{x}} \ll \frac{1}{\epsilon},
$$

which establishes a relation between the time scale of the fast motion and the exit rate/time from metastable subsets in the fast DOF, does not hold, then deviations from the averaged dynamics may be relevant.

Remark. It follows from (4.8) that $\partial_{t} \Pi \rho_{1}=\overline{\mathcal{L}} \Pi \rho_{1}+\Pi \mathcal{L}_{y}\left(\rho_{1}-\Pi \rho_{1}\right)$. This yields an equation of motion for $\Pi \rho_{1}$ since by our previous findings $\left(\rho_{1}-\Pi \rho_{1}\right)=\mathcal{L}_{x}^{-1}\left(\overline{\mathcal{L}}-\mathcal{L}_{y}\right) \rho_{0}$. If we denote the semigroup generated by $\overline{\mathcal{L}}$ by $\exp (t \overline{\mathcal{L}})$, then $\Pi \rho_{1}$ can be expressed as

$$
\Pi \rho_{1}(t)=e^{t \overline{\mathcal{L}}} \Pi \rho_{1}(t=0)+\int_{0}^{t} e^{(t-s) \overline{\mathcal{L}}} \Pi \mathcal{L}_{y} \mathcal{L}_{x}^{-1}\left(\overline{\mathcal{L}}-\mathcal{L}_{y}\right) \rho_{0}(s) d s .
$$

Thus, the first order contribution to the evolution of the $\Pi$-averaged density, $\Pi \rho_{1}$, also is in danger to become significantly large (in the sense that $\epsilon \Pi \rho_{1}$ is comparable to $\left.\Pi \rho_{0}\right)$ if $\delta_{x}$ is as small (or smaller) as $\epsilon$.

In the following we aim at cases where $\delta_{x}$ is of the order of $\epsilon$ such that the standard averaging scheme is in risk to fail. Then we expect that the original dynamics exhibits three scales: the fast scale of motion in $y$, the slow scale of motion in $x$, and the (comparable) scale of rare transitions between metastable sets in $y$ (if the gap $\delta_{x}$ is $\mathcal{O}(\epsilon)$, then the transitions in $y$ happen on time scales $\mathcal{O}(1) !)$.

5. Conditional averaging. Whenever $\epsilon>0$ is small but not small compared to the minimal spectral gap $\delta_{x}$, averaging might not be appropriate to provide a good reduced model for the slow dynamics. We will now show that we can construct an appropriate reduced model under the following assumption, which is the analogue formulation of the general Assumption 1.

Assumption 2. There is a small positive integer $M$ and some positive radius $R \gg \epsilon$ such that for every $x$ the intersection of the $L^{2}\left(\mu_{x}\right)$-spectrum of $\mathcal{L}_{x}$ with the interval $[-R, 0]$ is discrete and contains at most $M$ isolated eigenvalues $0=\lambda_{0}(x)>$ $\lambda_{1}(x) \geq \cdots \geq \lambda_{m(x)}(x)$, with $m(x)<M$. 
Remark. We will preliminarily ignore the problem that for some $x$ the number of dominant eigenvalues $m(x)+1$ may be smaller than $M$. Instead we will assume that there are exactly $M$ eigenvalues in the dominant spectrum for all $x$. More precisely, if we really have to deal with $m(x)+1<M$, we will assume that there are additional isolated eigenvalues $\lambda_{k}(x), k=m(x)+2, \ldots, M-1$, that play the role needed here but are not contained in the dominant spectrum since $\lambda_{k}(x)<-R$.

Let $u_{0}(x, \cdot)=\mathbf{1}, u_{1}(x, \cdot), \ldots, u_{M-1}(x, \cdot)$ denote the eigenvectors associated with the eigenvalues $\lambda_{0}, \ldots, \lambda_{M-1}$ in $L^{2}\left(\mu_{x}\right)$, i.e.,

$$
\mathcal{L}_{x} u_{k}(x, \cdot)=\lambda_{k}(x) u_{k}(x, \cdot) \text { in } L^{2}\left(\mu_{x}\right) .
$$

5.1. Motivation. We observed that deviations from the averaged dynamics have to be anticipated if the spectral gap $\delta_{x}$ of $\mathcal{L}_{x}$ w.r.t. its lowest eigenvalue $\lambda=0$ in $L^{2}\left(\mu_{x}\right)$ is comparable to the scale $\epsilon$. If we want to study this effect by means of multiscale asymptotics we have to explicitly couple $\delta_{x}$ to $\epsilon$. In order to motivate our further approach, we therefore assume that $\lambda_{k}=\epsilon \tilde{\lambda}_{k}, k=0, \ldots, M-1$, with $\tilde{\lambda}_{k}=\mathcal{O}(1)$, while the reminder of the spectrum is left unchanged, i.e., contributes to order $\mathcal{O}(1)$ only. Explicitly,

$$
\sigma\left(\mathcal{L}_{x}\right) \subset\left\{0, \epsilon \tilde{\lambda}_{1}, \ldots, \epsilon \tilde{\lambda}_{M-1}\right\} \cup[-R,-\infty) .
$$

Remark. The assumption (5.1) represents a modeling step: The dominant eigenvalues of $\mathcal{L}_{x}$ scale like $\exp \left(-\beta \Delta V_{i}^{x}\right)$, with $\Delta V_{i}^{x}$ denoting the most important energy barriers in the system for fixed $x$. Thus, a situation like (5.1) can explicitly be realized only if these barriers scale like $\Delta V_{i}^{x} \propto-\log \epsilon$. However, in this case the corresponding eigenvectors will also depend on $\epsilon$, an effect that fortunately contributes to higher orders only (see the remark below (5.7)). The detailed discussion of the effects of such a scaling of the main potential energy barriers is a lengthy procedure and will not be included herein but in a forthcoming paper.

As a consequence of (5.1) we can express $\mathcal{L}_{x}$ in the following form:

$$
\mathcal{L}_{x}=\epsilon \sum_{k=0}^{M-1} \tilde{\lambda}_{k}(x)\left\langle u_{k}, \cdot\right\rangle_{\mu_{x}} u_{k}+\tilde{R}_{x}=\epsilon \mathcal{L}_{x}^{\mathrm{act}}+\tilde{R}_{x},
$$

with $\tilde{R}_{x} u_{k}=0$ for all $k=0, \ldots, M-1$.

As we have seen, averaging can be understood as the projection of the dynamics onto the lowest eigenvalue that is associated with the invariant density in the $y$-direction; this projection had the form

$$
(\Pi f)(x)=\langle\mathbf{1}, f\rangle_{\mu_{x}} \mathbf{1},
$$

where 1 is the eigenvector associated with $\lambda_{0}(x)=0$ for all $x$. In the case considered now the simple idea is to project the dynamics onto the subspace spanned by the dominant spectrum, i.e., by the $M$ isolated dominant eigenmodes,

$$
S^{\text {act }}=\operatorname{span}\left\{u_{k}, k=0, \ldots, M-1\right\} .
$$

Thus, the projection is now given by

$$
(\tilde{\Pi} f)(x)=\sum_{k=0}^{M-1}\left\langle u_{k}, f\right\rangle_{\mu_{x}} u_{k} .
$$


We obviously again have $\mathcal{L}_{x} \tilde{\Pi}=\tilde{\Pi} \mathcal{L}_{x}$, but this time we do not have $\tilde{\Pi} \mathcal{L}_{x}=0$ too but only

$$
\tilde{R}_{x} \tilde{\Pi}=\tilde{\Pi} \tilde{R}_{x}=0 .
$$

In terms of the multiscale asymptotics of section 4.2 the basic idea of conditional averaging is to repeat the procedure of section 4.2 and observe some important differences: The lowest order $\left(\epsilon^{-1}\right)$ now yields $\tilde{R}_{x} \rho^{0}=0$; that is, $\rho^{0}$ satisfies

$$
\tilde{\Pi} \rho^{0}=\rho^{0} .
$$

The next order yields

$$
\tilde{R}_{x} \rho^{1}+\mathcal{L}_{y} \rho^{0}+\mathcal{L}_{x}^{\text {act }} \rho^{0}=\partial_{t} \rho^{0},
$$

which, after application of $\tilde{\Pi}$, gives us

$$
\partial_{t} \rho^{0}=\left(\tilde{\Pi} \mathcal{L}_{y} \tilde{\Pi}+\mathcal{L}_{x}^{\text {act }}\right) \rho^{0},
$$

the explicit form of which is evaluated in the next subsections. In addition, measuring the deviation from the full dynamics gives an indicator for its reliability that contains the spectral gap $\tilde{\delta}_{x} \geq R$ between $\lambda_{0}=0$ and the remainder of the spectrum (if Assumption 2 holds, it guarantees $\tilde{\delta}_{x} \geq R$ such that the indicator would always indicate the validity of the procedure).

5.2. Projection onto dominant subspace. The previous motivation tells us that the projection onto the dominant subspace yields a reduced dynamical model (to be derived from its Fokker-Planck equation (5.4)) that will have a rigorous justification under the scaling assumption (5.1). However, let us for the moment forget about this assumption and (simply) evaluate the consequences of projecting the full FokkerPlanck equation (2.1) onto the subspace associated with the dominant spectrum. In order to do so we consider the ansatz

$$
\rho^{\epsilon}(x, y, t)=\sum_{k=0}^{M-1} c_{k}(x, t) u_{k}(x, y) .
$$

In Appendix B we show how this ansatz, if inserted into the Fokker-Planck equation (2.1), yields $M$ independent evolution equations for the coefficients $c_{k}$ :

$$
\begin{gathered}
\partial_{t} c_{0}=\overline{\mathcal{L}} c_{0}+\sum_{k=1}^{M-1}\left(\left\langle\mathbf{1}, \mathcal{L}_{y} u_{k}\right\rangle_{\mu_{x}} c_{k}-\left\langle u_{k}, D_{x} V\right\rangle_{\mu_{x}} \cdot D_{x} c_{k}\right. \\
\left.+\sigma^{2}\left\langle\mathbf{1}, D_{x} u_{k}\right\rangle_{\mu_{x}} \cdot D_{x} c_{k}\right),
\end{gathered}
$$

and, for $k>0$,

$$
\begin{array}{r}
\partial_{t} c_{k}=\tilde{\mathcal{L}}_{k} c_{k}+\sum_{\substack{l=0 \\
l \neq k}}^{M-1}\left(\left\langle u_{k}, \mathcal{L}_{y} u_{l}\right\rangle_{\mu_{x}} c_{l}-\left\langle u_{k}, u_{l} D_{x} V\right\rangle_{\mu_{x}} \cdot D_{x} c_{l}\right. \\
\left.+\sigma^{2}\left\langle u_{k}, D_{x} u_{l}\right\rangle_{\mu_{x}} \cdot D_{x} c_{l}\right)+\frac{1}{\epsilon} \lambda_{k} c_{k},
\end{array}
$$

with $\tilde{\mathcal{L}}_{k}$ given by (B.2). We now can distinguish two cases: 
(1) If we now assume the spectrum obeys the scaling according to (5.1) we obtain the explicit form of (5.4) by replacing each $\lambda_{k} / \epsilon$ by $\tilde{\lambda}_{k}$ in the evolution equations (5.6).

(2) In contrast, assume that the dominant eigenvalues do not scale with $\epsilon$, i.e., $\lambda_{k}=\mathcal{O}(1)$. Now, careful inspection reveals that for sufficiently small $\epsilon$ the damping term $c_{k} \lambda_{k} / \epsilon<0$ in (5.6) grows, and therefore the $c_{k}$ vanish, except for $k=0$. Then the standard averaged Fokker-Planck equation $\partial_{t} c_{0}=\overline{\mathcal{L}} c_{0}$ turns out to be a special case of the current scheme.

Unfortunately, the form of the above evolution equations does not allow for a lucid interpretation or for some simple simulation scheme that could efficiently be applied to higher dimensions. Therefore, in the next step we will construct an alternative formulation that models the evolution of the coefficients only approximately but allows for an intriguing interpretation and direct numerical simulation.

5.3. Derivation of conditional averaging in explicit form. For ease of derivation let us assume that Assumption 2 is satisfied for $M=2$. Then, for each fixed $x$, the zero of the second eigenvector $u_{1}$ of $\mathcal{L}_{x}$ in $L^{2}\left(\mu_{x}\right)$ decomposes the fiber $\Phi(x)$ according to

$$
B_{1}=B_{x}=\left\{y: u_{1}(x, y)<0\right\} \text { and } B_{2}=B_{x}^{c}=\left\{y: u_{1}(x, y) \geq 0\right\} .
$$

Let us denote the indicator functions of $B_{x}$ and $B_{x}^{c}$ by

$$
\chi_{1}=\mathbf{1}_{B_{1}}=\mathbf{1}_{B_{x}}, \quad \chi_{2}=\mathbf{1}_{B_{2}}=\mathbf{1}_{B_{x}^{c}} \Rightarrow \chi_{1}+\chi_{2}=\mathbf{1}(x, \cdot) .
$$

Now, remember that due to (3.2) $u_{1}$ is an approximate step-function:

$$
u_{1}(x, \cdot) \approx \sqrt{\frac{\mu_{x}\left(B_{2}\right)}{\mu_{x}\left(B_{1}\right)}} \chi_{1}-\sqrt{\frac{\mu_{x}\left(B_{1}\right)}{\mu_{x}\left(B_{2}\right)}} \chi_{2}=\tilde{u}_{1} .
$$

Remark. The results of [13], e.g., guarantee that $\left\|u_{1}-\tilde{u}_{1}\right\|_{\mu_{x}}=\mathcal{O}\left(\epsilon^{1 / 2}\right)$ if the dominant spectrum satisfies (5.1) for $M=2$. However, these results hold for arbitrary Markov semigroups that are generated by nonpositive self-adjoint operators in the appropriately weighted Hilbert space. The additional structure of the case under consideration (especially the specific form of the weighted norm) allows significantly stronger results: For example, if for given $x$ the most important barrier $\Delta V_{x}$ in the potential energy scales like $\Delta V_{x} \propto-\ln \epsilon$, then $\left\|u_{1}-\tilde{u}_{1}\right\|_{\mu_{x}}=\mathcal{O}(\epsilon)$; see, e.g., Theorem 5.4 .2 of [44].

For the next steps we will proceed as if we had equality in (5.7). Then

$$
\left\langle u_{1}, \chi_{1}\right\rangle_{\mu_{x}}=-\left\langle u_{1}, \chi_{2}\right\rangle_{\mu_{x}}=\gamma=\sqrt{\mu_{x}\left(B_{1}\right) \mu_{x}\left(B_{2}\right)} .
$$

Therefore

$$
\begin{aligned}
& \chi_{1}=\mu_{x}\left(B_{1}\right) \mathbf{1}+\gamma u_{1}, \\
& \chi_{2}=\mu_{x}\left(B_{2}\right) \mathbf{1}-\gamma u_{1} .
\end{aligned}
$$

If (3.2) holds exactly, $\left\{\chi_{1}, \chi_{2}\right\}$ is a basis of the dominant subspace of $L^{2}\left(\mu_{x}\right)$ spanned by the two dominant eigenvectors. Thus we can reformulate our ansatz (5.5) in the following form:

$$
\rho^{\epsilon}(x, y, t)=c_{1}(x, t) \chi_{1}(x, y)+c_{2}(x, t) \chi_{2}(x, y) .
$$


Inserting this into the Fokker-Planck equation yields

$$
\begin{aligned}
& \mu_{x}\left(B_{1}\right) \partial_{t} c_{1}=\left\langle\chi_{1}, \mathcal{L}_{y} \rho^{\epsilon}\right\rangle_{\mu_{x}}+\frac{1}{\epsilon} \gamma^{2} \lambda_{1}\left(c_{1}-c_{2}\right), \\
& \mu_{x}\left(B_{2}\right) \partial_{t} c_{2}=\left\langle\chi_{2}, \mathcal{L}_{y} \rho^{\epsilon}\right\rangle_{\mu_{x}}+\frac{1}{\epsilon} \gamma^{2} \lambda_{1}\left(c_{2}-c_{1}\right) .
\end{aligned}
$$

Evaluation of the scalar products on the right-hand side (RHS) of these equations leads to

$$
\begin{aligned}
& \left\langle\chi_{1}, \mathcal{L}_{y} \rho^{\epsilon}\right\rangle_{\mu_{x}}=\frac{\sigma^{2}}{2} \mu_{x}\left(B_{1}\right) \Delta_{x} c_{1}-\left\langle\chi_{1}, D_{x} V\right\rangle_{\mu_{x}} \cdot D_{x} c_{1}+\text { B.T. } \\
& \left\langle\chi_{2}, \mathcal{L}_{y} \rho^{\epsilon}\right\rangle_{\mu_{x}}=\frac{\sigma^{2}}{2} \mu_{x}\left(B_{2}\right) \Delta_{x} c_{2}-\left\langle\chi_{2}, D_{x} V\right\rangle_{\mu_{x}} \cdot D_{x} c_{2}+\text { B.T. }
\end{aligned}
$$

where B.T. stands for boundary terms that depend on the boundary between $B_{1}=B_{x}$ and $B_{2}=B_{x}^{c}$ for different $x$. These terms are ignored in the following and will be discussed in Appendix A. In order to compute the remaining terms, it is reasonable to define two additional measures for each fixed value of $x$. These correspond to the densities

$$
\mu_{x}^{(j)}(y)=\frac{1}{\mu_{x}\left(B_{j}\right)} \mu_{x}(y) \chi_{j}(y), \quad j=1,2 .
$$

By construction, $\mu_{x}^{(1)}$ is supported on $B_{1}$ only, while $\mu_{x}^{(2)}$ is supported on the complement $B_{2}$. With this notation we define two different averaged generators, $j=1,2$ :

$$
\begin{aligned}
\overline{\mathcal{L}}^{(j)} & =\frac{\sigma^{2}}{2} \Delta_{x}-\left\{\int D_{x} V \mu_{x}^{(j)}(y) \mathrm{d} y\right\} \cdot D_{x} \\
& =\frac{\sigma^{2}}{2} \Delta_{x}-D_{x} \bar{V}^{(j)} \cdot D_{x}
\end{aligned}
$$

where the two averaged potentials are given by

$$
\bar{V}^{(j)}(x)=-\frac{1}{\beta} \log \int_{B_{j}} \exp (-\beta V(x, y)) \mathrm{d} y, \quad j=1,2 .
$$

Consequently, the evolution equations for $c_{1}$ and $c_{2}$ read

$$
\begin{aligned}
& \partial_{t} c_{1}=\overline{\mathcal{L}}^{(1)} c_{1}+\frac{1}{\epsilon} \mu_{x}\left(B_{2}\right) \lambda_{1}\left(c_{1}-c_{2}\right), \\
& \partial_{t} c_{2}=\overline{\mathcal{L}}^{(2)} c_{2}+\frac{1}{\epsilon} \mu_{x}\left(B_{1}\right) \lambda_{1}\left(c_{2}-c_{1}\right) .
\end{aligned}
$$

5.4. Discretization and interpretation. This section will discuss the construction of some simple discretization scheme for the evolution equations (5.12) and (5.13). The authors want to emphasize that it is not their goal to construct a discretization here that may satisfy the standards of numerical mathematics. For the scope of the present paper we rather are interested in a "quick and dirty version" that (a) allows us to develop a lucid interpretation of these equations and that (b) allows for pathwise simulation without the need to discretize the evolution equations as partial differential equations. 
To this end, we introduce the vector $c=\left(c_{1}, c_{2}\right)^{T}$ and rewrite the evolution equations (5.12) and (5.13) as

$$
\begin{aligned}
\partial_{t} c=\left(\bar{£}+\overline{\mathcal{R}}_{x}\right) c, \quad \text { with } \quad \bar{£} & =\left(\begin{array}{cc}
\overline{\mathcal{L}}^{(1)} & 0 \\
0 & \overline{\mathcal{L}}^{(2)}
\end{array}\right), \\
\text { and } \quad \overline{\mathcal{R}}_{x} & =\frac{\left|\lambda_{1}\right|}{\epsilon}\left(\begin{array}{cc}
-\mu_{x}\left(B_{2}\right) & \mu_{x}\left(B_{2}\right) \\
\mu_{x}\left(B_{1}\right) & -\mu_{x}\left(B_{1}\right)
\end{array}\right) .
\end{aligned}
$$

This equation is now discretized in time by means of some simple splitting scheme, e.g., by the Trotter scheme, which yields (in the usual somewhat sloppy notation)

$$
c(\tau, \cdot)=\exp \left(\tau\left(\bar{£}+\overline{\mathcal{R}}_{x}\right)\right) c(t=0, \cdot)=\exp \left(\tau \overline{\mathcal{R}}_{x}\right) \exp (\tau \bar{£}) c(t=0, \cdot)+\mathcal{O}\left(\tau^{2}\right),
$$

where $\tau$ (unfortunately) has to be of order $o(\epsilon)$. The advantage of this discretization is that $\exp (\tau \bar{£})$ has a direct pathwise realization since it denotes the time- $\tau$ transport of the Fokker-Planck equations associated with the two SDEs:

$$
\dot{x}=-D_{x} \bar{V}^{(j)}(x)+\sigma \dot{W}, \quad j=1,2 .
$$

The second term, $\exp \left(\tau \overline{\mathcal{R}}_{x}\right)$, obviously denotes a "simple" exchange between the two levels $j=1$ and $j=2$ :

$$
\exp \left(\tau \overline{\mathcal{R}}_{x}\right)=\left(\begin{array}{cc}
1+\mu_{x}\left(B_{2}\right)\left(e^{\tau \lambda_{1} / \epsilon}-1\right) & -\mu_{x}\left(B_{2}\right)\left(e^{\tau \lambda_{1} / \epsilon}-1\right) \\
-\mu_{x}\left(B_{1}\right)\left(e^{\tau \lambda_{1} / \epsilon}-1\right) & 1+\mu_{x}\left(B_{1}\right)\left(e^{\tau \lambda_{1} / \epsilon}-1\right)
\end{array}\right) .
$$

This matrix is $x$-dependent through the $\mu_{x}\left(B_{j}\right)$ and $\lambda_{1}=\lambda_{1}(x)$. Nevertheless, it is a stochastic matrix for all $\tau \geq 0$. Therefore, one step of the Trotter discretization $c(t+\tau, \cdot)=\exp \left(\tau \overline{\mathcal{R}}_{x}\right) \exp (\tau \overline{\bar{£}}) c(t, \cdot)$ has a pathwise realization within a specific stochastic particle method (cf. [28]):

Let $x^{(j)}(t)$ denote the positions of an ensemble of particles with numbers $j=1, \ldots, N$ and level numbers $l_{j} \in\{1,2\}, j=1, \ldots, N$, at time $t$. Assume that this ensemble represents the initial state $c(t, \cdot)$ in the following way: The subsequence $x^{(j)}(t)$ with $l_{j}=1$ is a sampling of the probability density function $c_{1}(t, \cdot)$, and the subsequence $x^{(j)}(t)$ with $l_{j}=2$ is a sampling of $c_{2}(t, \cdot)$. Realization of the Trotter discretization then requires two steps:

Step 1 (transport). Determine for each of the particles an updated position $x^{(j)}(t+\tau)$ by solving

$$
\dot{x}=-D_{x} \bar{V}^{\left(l_{j}\right)}(x)+\sigma \dot{W}, \quad x(t=0)=x^{(j)}(t),
$$

over $[0, \tau]$.

Step 2 (exchange). For each particle on level $j=1$ (i.e., $l_{j}=1$ ), set $l_{j}=2$ with hopping probability $p_{1 \rightarrow 2}=\mu_{x}\left(B_{2}\right)\left(1-e^{\tau \lambda_{1} / \epsilon}\right)$ and remain at $l_{j}=1$ with probability $1-p_{1 \rightarrow 2}$. For each particle on level $j=2$, set $l_{j}=1$ with probability $p_{2 \rightarrow 1}=\mu_{x}\left(B_{1}\right)\left(1-e^{\tau \lambda_{1} / \epsilon}\right)$ and remain at $l_{j}=2$ with probability $1-p_{2 \rightarrow 1}$.

The resulting ensemble then represents $c(t+\tau, \cdot)$ in the same sense as it previously did for $c(t, \cdot)$. Hence, iteration of this procedure means simulation of the evolution equations of our conditional averaging scheme. 
6. Illustrative examples. In this section we will illustrate the results from the preceding sections by numerical experiments with appropriate test examples.

6.1. Averaging appropriate. We first are interested in the relation between the validity of the averaging scheme as an approximate, reduced model with

- the approximate constancy of the dominant eigenvectors in the $y$-direction,

- the existence of a significantly large spectral gap $\delta_{x}$.

First of all we consider the potential

$$
V(x, y)=\frac{1}{2}\left(x^{2}-1\right)^{2}+\frac{1}{2} \omega(x)^{2} y^{2},
$$

which is nothing more than a double well potential in the $x$-direction and a harmonic potential in the $y$-direction. Both are coupled by the $x$-dependence of the harmonic constant $\omega(x)^{2}$. However, we choose $\omega$ such that it exhibits some small oscillations around the constant value 1 mostly but significantly deviates from 1 in the vicinity of $x_{p}=1$ :

$$
\omega(x)=\frac{3}{2}+\frac{1}{2} \sin (5 \pi / 2 x)+\kappa \exp \left(-\Sigma(x-1)^{2}\right), \quad \kappa=75, \quad \Sigma=185 .
$$

Figures 1 and 2 show the form of $\omega$, the full potential, and the corresponding averaged potential. We observe that the peak at $x_{p}=1$ induces an additional barrier in the averaged potential. This illustrates the case of so-called dynamical barriers that are induced by collective effects of the fast coordinates only.

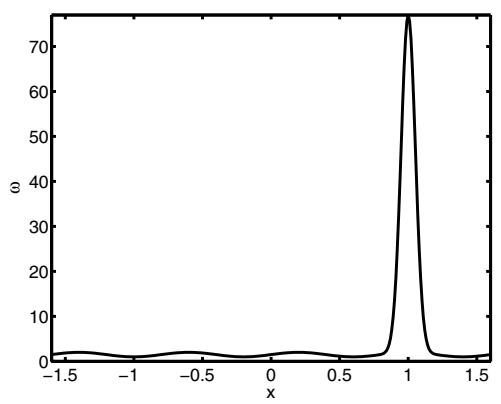

FIG. 1. Form of $\omega=\omega(x)$ used herein.
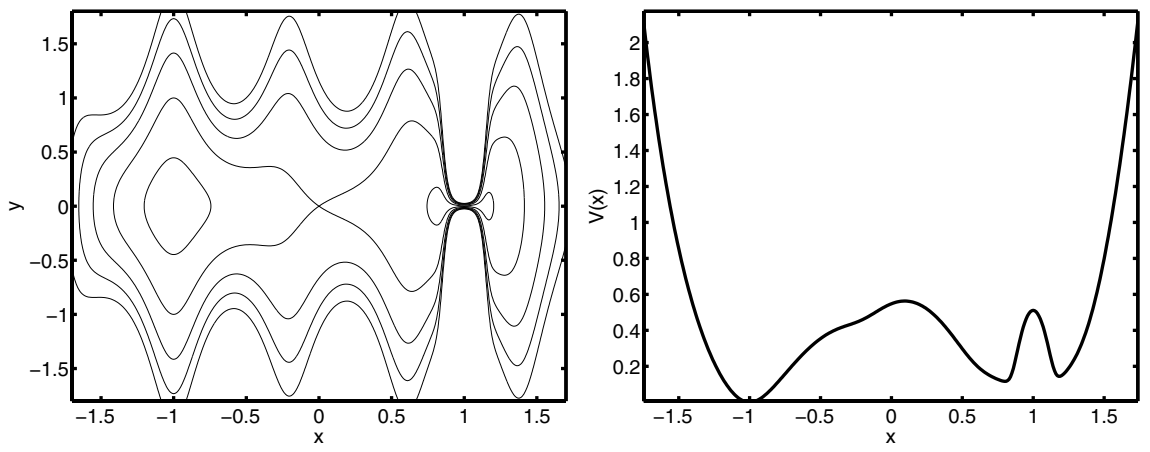

FIG. 2. Full and averaged potential for $\beta=8.5$. 
TABLE 1

First four eigenvalues of the full generator $\mathcal{L}^{\epsilon}$ and the averaged one $\overline{\mathcal{L}}$. The gap between $\lambda_{2}$ and $\lambda_{3}$ indicates three metastable sets.

\begin{tabular}{|c|c|c|c|c|}
\hline Eigenv. & $\lambda_{0}$ & $\lambda_{1}$ & $\lambda_{2}$ & $\lambda_{3}$ \\
\hline $\mathcal{L}^{\epsilon}$ & 0.000 & -0.016 & -0.290 & -1.527 \\
\hline$\overline{\mathcal{L}}$ & 0.000 & -0.016 & -0.323 & -1.527 \\
\hline
\end{tabular}

If we choose $\epsilon=0.1$ and if $\beta=8.5$, the dominant eigenvalues of the full and averaged generators, $\mathcal{L}^{\epsilon}$ and $\overline{\mathcal{L}}$, are shown in Table 1 .

Figures 3 and 4 show the dominant eigenvectors of the full generator for these parameters, their projected versions, and the corresponding eigenvectors of the averaging dynamics. For all values of $x$ the spectral gap $\delta_{x}$ is essentially larger than $\epsilon$ : it takes values around 1 and even larger values near $x_{p}=1$.
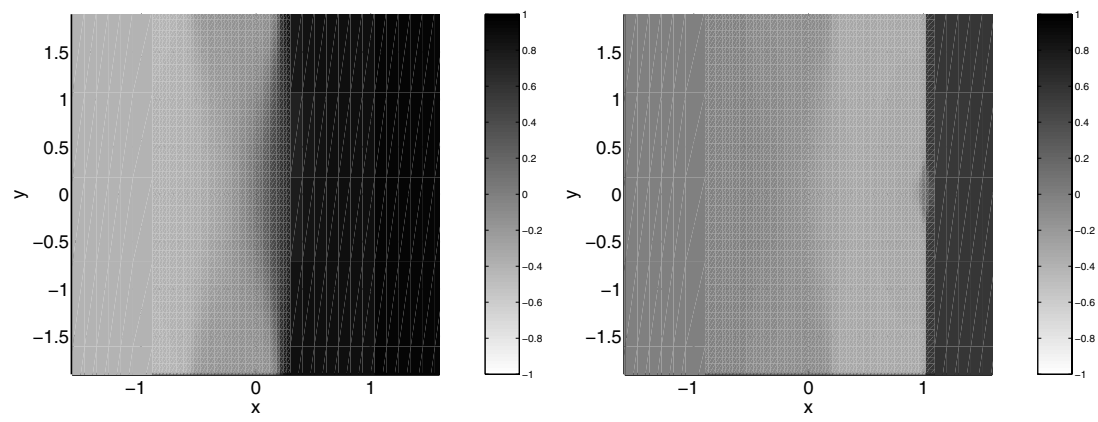

Fig. 3. Second and third eigenvector of full potential for $\epsilon=0.1$. There are only minor deviations from almost constancy in the $y$-direction.
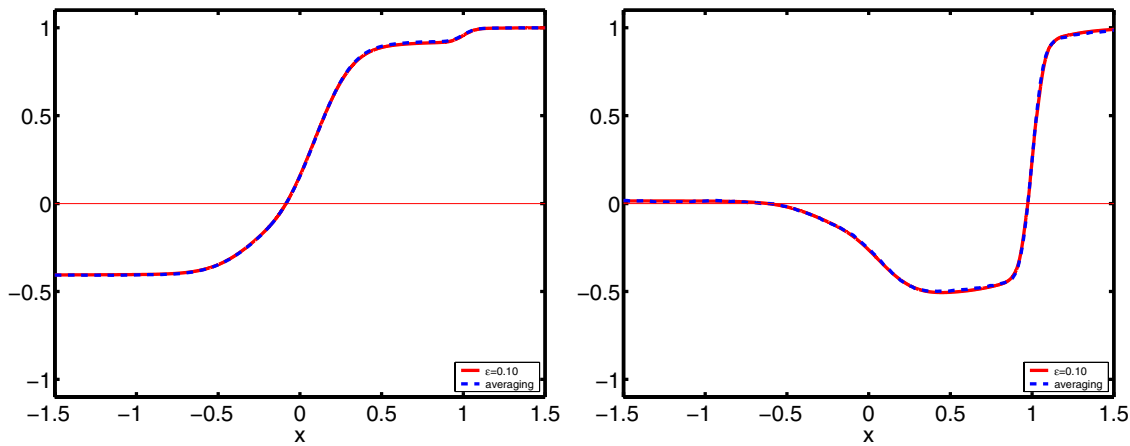

FIG. 4. Second and third eigenvector of averaged potential (solid) and projections $\Pi u_{1}$ and $\Pi u_{2}$ of second and third eigenvector $u_{1}$ and $u_{2}$ of full potential $(\epsilon=0.1, \beta=8.5)$.

Note that, for fixed $x$, the stochastic process on each fiber is of Ornstein-Uhlenbeck type. Therefore, explicit expressions for the eigenvalues of $\mathcal{L}_{x}$ are known: For example, the first eigenvalue is $\lambda_{1}(x)=-\omega(x)^{2}$, which due to the present choice of $\omega$ is always well separated from $\lambda_{0} \equiv 0$.

According to our theory, this should indicate that the averaging scheme will yield an appropriately reduced model. In Figure 5 we compare a realization of the averaged motion to the full motion, which confirms our conjecture. 

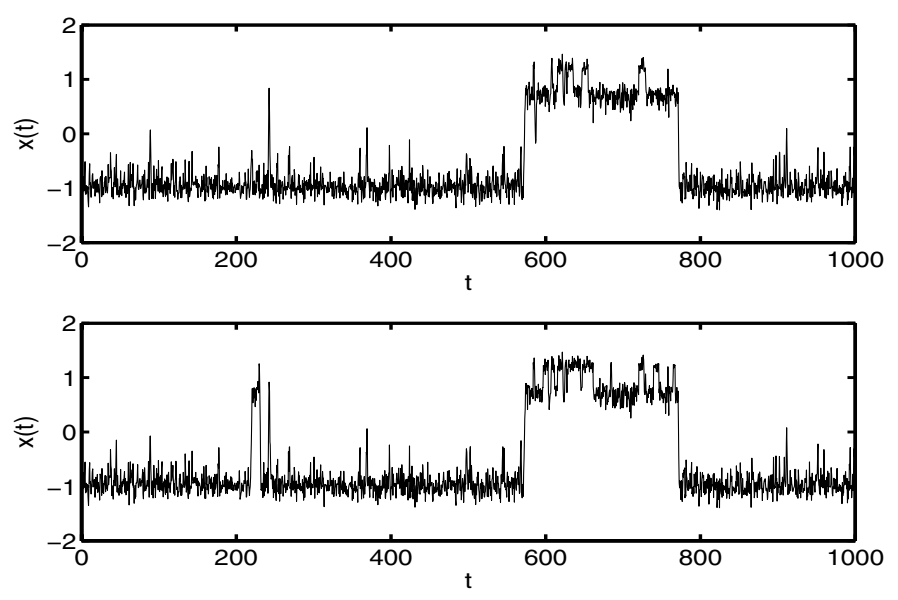

FIG. 5. Comparison of a typical realization of the full dynamics' $x$-coordinate (top) to the averaged motion (bottom). The realizations have been computed for one realization of the white noise.

6.2. Averaging inappropriate. In order to illustrate the problems that may arise in averaging, we now consider the following potential:

$$
V(x, y)=5 \cdot\left(y^{2}-1\right)^{2}+1.25 \cdot(y-x / 2)^{2} .
$$

The potential energy surface is shown in Figure 6 together with the corresponding averaged potential.
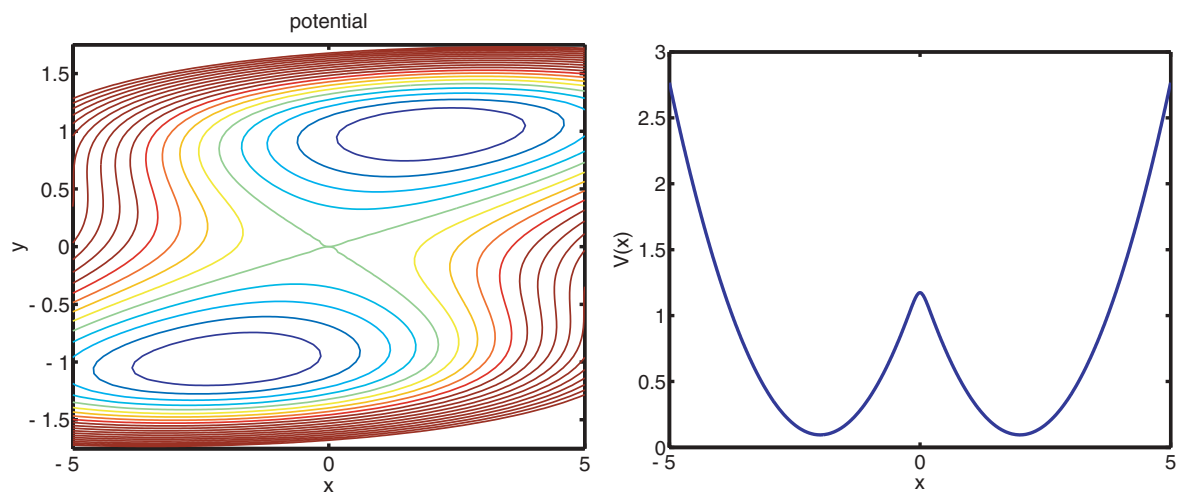

FIG. 6. Full and averaged potential for $\beta=8.5$.

We consider $\epsilon=0.005$; i.e., diffusion in $y$ is very fast compared to diffusion in $x$. However, the important barriers of the potential are barriers in the $y$-direction; see Figure 6 . Thus for fixed $\epsilon$, increasing the inverse temperature $\beta$ increases the metastability in $y$. Consequently, by choosing different $\beta$ one can analyze the effect of increasing metastability on averaging. For $\epsilon=0.005$ and $\beta \geq 1$, Table 2 shows two eigenvalues near zero.

All other eigenvalues are significantly smaller than -1 . The second eigenvector is shown in Figure 7 for $\epsilon=0.005$ and $\beta=1.00,1.75,2.50$. One immediately observes that the eigenvectors are constant in the $y$-direction for $\beta=1.0$ only. Deviations grow 
TABLE 2

Dominant eigenvalues of the full generator $\mathcal{L}^{\epsilon}$ with $\epsilon=0.005$ for different temperatures. Metastability occurs as the temperature decreases.

\begin{tabular}{|c|c|c|c|}
\hline Eigenv. of $\mathcal{L}^{\epsilon}$ & $\beta=1.00$ & $\beta=1.75$ & $\beta=2.50$ \\
\hline$\lambda_{0}$ & 0.000 & 0.000 & 0.000 \\
\hline$\lambda_{1}$ & -0.160 & -0.050 & -0.003 \\
\hline
\end{tabular}
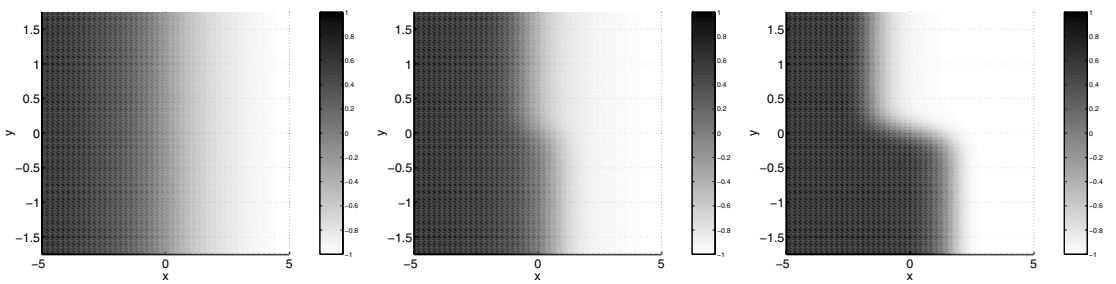

FIG. 7. Second eigenvectors of the full potential for $\epsilon=0.005$ and $\beta=1.0$ (left), $\beta=1.75$ (middle), and $\beta=2.5$ (right).

as $\beta$ becomes larger. From the pictures we may expect that for $\beta \leq 1.00$ averaging works pretty well, whereas it does not for larger $\beta$.

Figure 8 illustrates that the deviation from constancy has to do with a spectral gap $\delta_{x}$ taking values in the order of magnitude of $\epsilon$ : For $x$ in a neighborhood of $x=0$, $\delta_{x}$ comes close to $\epsilon$ for $\beta=1.75$ and is even smaller than $\epsilon$ for $\beta \geq 2.5$, while it is clearly bounded away from $\epsilon$ for $\beta=1$.0.

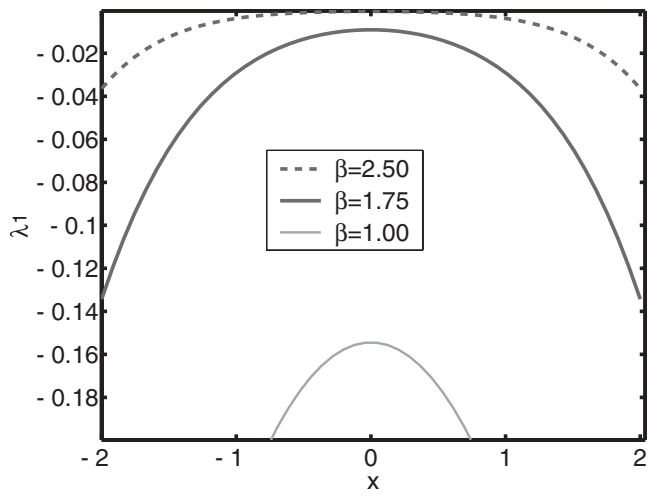

FiG. 8. Spectral gap $\delta_{x}$ for $\epsilon=0.005$.

In order to demonstrate the deviation between averaged and full dynamics, we first show typical realizations for $\beta=2.5$ and $\epsilon=0.005$ in Figure 9. The trajectories have been generated using the Euler-Maruyama scheme with fixed time step $\mathrm{d} t=0.001$ for the averaged dynamics and $\mathrm{d} t=\epsilon / 10$ for the full system, respectively. We found that the results are robust under changes of step-size. We clearly observe essential differences; in particular, the transition rate from $x<0$ to $x>0$ seems significantly smaller for the averaged case than for the full one.

In order to analyze this deviation more carefully, we consider the exit times from the set

$$
S=\{x: x<2\} .
$$



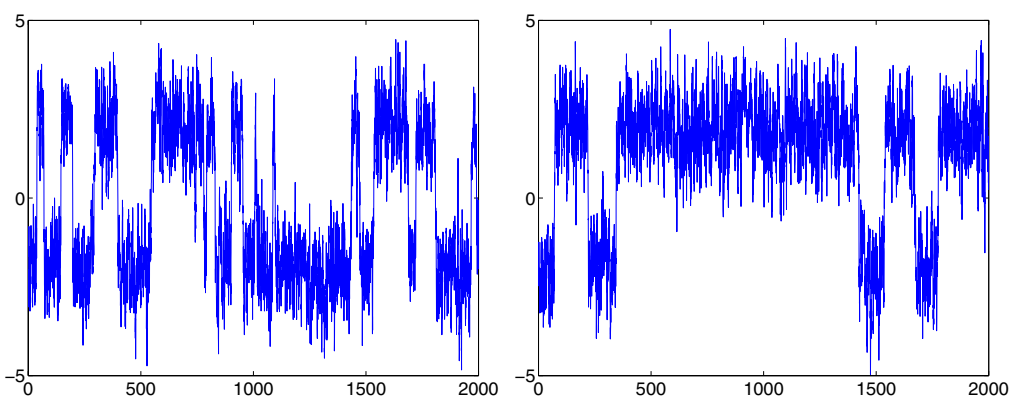

FIG. 9. Single realizations of the averaged (left) and the full (right) dynamics for $\beta=2.5$.

More exactly, we consider the random variable

$$
\varrho_{S}=\inf \left\{t>0: \int_{0}^{t} \mathbf{1}_{S^{c}}\left(x^{\epsilon}(s)\right) \mathrm{d} s>0 \mid x^{\epsilon}(0)=-2, y^{\epsilon}(0)=-1\right\},
$$

which measures the first time at which the process exits from $S$ and enters the complement $S^{c}{ }^{1}$ We estimate the expectation value of the first exit time by means of $N=2000$ realizations of both averaged dynamics and full dynamics. For $\epsilon=0.005$ we find significant deviations for larger values of $\beta$ in full agreement with the observation of Figures 7 and 8, as it is shown in Table 3 .

\section{TABLE 3}

Exit times from the set $S=\{x: x<2\}$ for standard averaging.

\begin{tabular}{|c|c|c|c|c|}
\hline Dynamical model & $\beta=1.00$ & $\beta=1.75$ & $\beta=2.00$ & $\beta=2.50$ \\
\hline $\mathbf{E}\left[\varrho_{S}\right]$ standard averaging & 16 & 32 & 40 & 63 \\
\hline $\mathbf{E}\left[\varrho_{S}\right]$ full dynamics & 16 & 40 & 70 & 404 \\
\hline
\end{tabular}

6.3. Conditional averaging. We now demonstrate the preeminence of conditional averaging for the previous case. To this end, we again inspect the long-term behavior of full dynamics and conditional averaging in Figure 10. Apparently, the transition rates from $x<0$ to $x>0$ now coincide to some extent.

However, in order to present numbers instead of pictures we again compute the expectation value of the exit times $\varrho_{S}$. From $N=2000$ realizations for $\epsilon=0.005$ we get a very good agreement between full dynamics and conditional averaging, as it is shown in Table 4 (in particular if we take into account the estimated statistical error of about $5 \%$ ).

Note that in contrast to the standard averaging procedure the simulations as shown in Figure 10 suggest that we can only expect convergence of the distributions instead of pathwise convergence.

Remark. In this example the number of isolated eigenvalues in the dominant spectrum of $\mathcal{L}_{x}$ is at most two for all $x$; i.e., Assumption 2 is satisfied for $M=2$. While for values of $x$ around $x=0$ there are definitely two dominant eigenvalues, Figure 8 illustrates that for large negative and positive values of $x$, say for $|x|>x_{\beta}$, there is

\footnotetext{
${ }^{1}$ The attentive reader may wonder why we define the set $S$ by $x<2$ rather than by $x<0$. The reason is that by $x<0$ we cannot adequately discriminate between the metastable sets corresponding to the two basins of attraction in the upper right and the lower left corner (cf. Figure 6). But whenever $x>2$ we can assume that most probably the system has left the lower basin.
} 

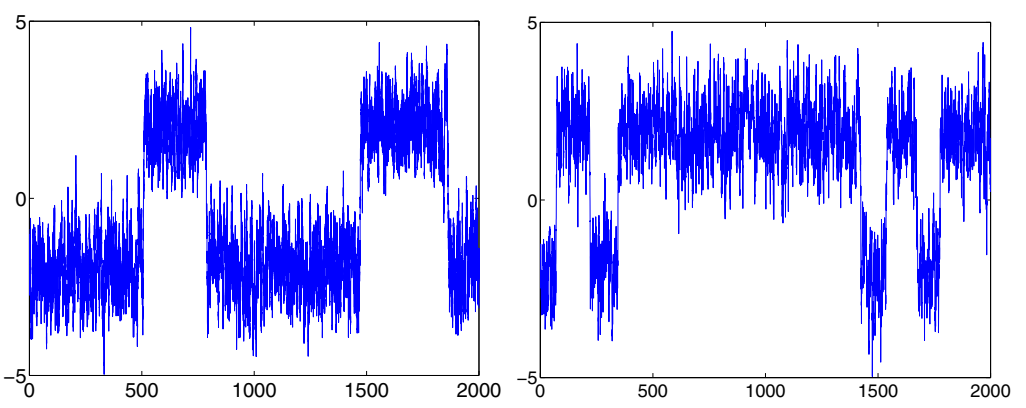

FIG. 10. Single typical realizations of the conditional averaging (left) and the full dynamics (right) for $\beta=2.5$.

TABLE 4

Exit times from the set $S=\{x: x<2\}$ for conditional averaging.

\begin{tabular}{|c|c|c|c|c|}
\hline Dynamical model & $\beta=1.00$ & $\beta=1.75$ & $\beta=2.00$ & $\beta=2.50$ \\
\hline $\mathbf{E}\left[\varrho_{S}\right]$ conditional averaging & 16 & 42 & 73 & 395 \\
\hline $\mathbf{E}\left[\varrho_{S}\right]$ full dynamics & 16 & 40 & 70 & 404 \\
\hline
\end{tabular}

only one dominant eigenvalue $(\lambda=0)$ with a large gap to the remaining spectrum. In view of our theoretical results, we conclude that the standard averaging scheme is appropriate for large negative and positive values of $x$, while the conditional averaging has to be used for values of $x$ around $x=0$. However, conditional averaging yields the same results as standard averaging whenever $\lambda=0$ is isolated by a large spectral gap (in this case the exchange process is not rare but frequent, and the mixing of the conditional averages reproduces the standard average). Therefore, conditional averaging can be used instead of standard averaging even for $|x|>x_{\beta}$. Consequently, whenever we want to compute a path of the reduced dynamics with an initial value $x<-x_{\beta}$, for example, we could think of two different algorithmic realizations: (1) We use the standard averaging scheme and switch to conditional averaging when entering the inner region $|x| \leq x_{\beta}$. The switch from standard averaging to conditional averaging is no problem since it is obvious in which of the wells of $V\left(x=-x_{\beta}, \cdot\right)$ we have to continue the path after entering from the left $\left(x<-x_{\beta}\right)$. However, it is likewise obvious that we can easily construct potentials $V$ for which the continuation problem at the switching point is significantly more complicated. (2) Alternatively, we simply use the conditional averaging scheme throughout the entire simulation (we did this for computing the realization shown in Figure 10). The advantage is obvious: We do not have to face any continuation problem. The disadvantage is that the switching process is fast compared to the slow motion in $x$. Improved discretization techniques for this case are subject to further investigation.

7. Molecular dynamics and related fields. The type of averaged equation of motion (4.9) is well known in molecular dynamics and materials science applications in the sense that the collective effect of the fast coordinates $y$ can be represented by means of the averaged potential

$$
\bar{V}(x)=-\frac{1}{\beta} \log Z_{x}, \quad Z_{x}=\int \exp (-\beta V(x, y)) .
$$

In these fields of application it is well known as conformational free energy potential or potential of means force $[34,35]$. However, it often is derived in a totally different, 
purely statistical way without any reference to dynamical concepts or to averaging or related techniques; see, e.g., [21] for a typical example.

Application to molecular dynamics? The setting of application to molecular dynamics may be sketched as follows: The transition path between some important initial and final states of the molecule under consideration is described in terms of the slow coordinates $x$ only; one averages over a vast number of fast coordinates (e.g., hundreds up to thousands of coordinates). The motion along the path is governed by the potential $\bar{V}$. However, the actual efficient evaluation of the integral defining $\bar{V}$ is a major challenge because of the curse of dimensionality. Therefore, one resorts to specific iterative sampling techniques, e.g., different Monte Carlo or constrained molecular dynamics techniques, diffusion or Langevin processes, to compute the integral $Z_{x}$, the averaged potential, or the averaged forces for some relevant values of $x$; cf. $[24,25,53]$. These techniques are designed to explore the accessible state space for fixed $x$ efficiently.

However, if the accessible state space contains significantly large energy barriers that separate some major potential wells from each other, then the sampling iteration typically gets trapped inside these wells. Then one needs a large number of iterations to escape from one well, which, in turn, critically slows down the exploration process. In most articles about state of the art sampling schemes most of the intellectual energy is invested to overcome this trapping problem or critical slowing down.

Dealing with the topic of this paper, it is of major importance that sampling schemes are amenable that overcome the trapping problem by means of identification of metastable sets exploiting the dominant spectrum of the transfer operator (or generator) of the dynamics $[19,20]$ in the course of iterating the sampling scheme. This implies that with such techniques the inevitable computation of $Z_{x}$ or the averaged forces produces exactly the information that is needed to replace averaging by conditional averaging if necessary. Then the application of conditional averaging produces only slightly more computational effort than the application of standard averaging. In forthcoming papers exactly this opportunity will be investigated.

Relation to materials science. The same basic problem (reliable extensive sampling of the accessible state space normal to the coordinates of major interest) emerges in the most recent approaches to transition path computation $[4,17]$ in materials science, molecular dynamics, and phase transition theory. Concepts and algorithms considered therein may serve as starting points to generalize the conditional averaging approach towards situations where the fast variable are normal directions of a nonflat slow manifold.

8. Conclusion. The article deals with averaging and related asymptotic techniques for the elimination of fast DOF in (stochastic) dynamical systems. It is motivated by the observation that the available techniques can be inappropriate if the fast DOF induce very long transition time scales due to metastability effects. These cases may appear in real-life applications, e.g., in molecular dynamics applications with fast torsion angle dynamics. We have derived indicators for the possible inadequacy of the averaging scheme for simple diffusion processes and have constructed an extension of the averaging technique for cases where the fast modes cause the longest time scales in the system. The authors' conjecture is that this construction is quite general: Its basic idea, the projection of the fast dynamics onto the dominant eigenspace of the transfer operator associated with the dynamics instead of projection onto the principal eigenmode (invariant measure) only, should be applicable to other classes of dynamical systems (even to deterministic scenarios; see the work of Dellnitz and Junge [14]). 
Appendix A. Boundary terms in conditional averaging. The boundary terms B.T. in the evolution equations of the conditional averaging scheme are related to the dependence of the boundary between $B_{1}=B_{x}$ and $B_{2}=B_{x}^{c}$ on $x$. For example, the B.T. in (5.9) are given by

$$
\text { B.T. }=\sum_{j=1}^{2}\left(\left\langle\chi_{1}, \mathcal{L}_{y} \chi_{j}\right\rangle_{\mu_{x}} c_{j}+\sigma^{2}\left\langle\chi_{1}, D_{x} \chi_{j}\right\rangle_{\mu_{x}} \cdot D_{x} c_{j}\right) .
$$

Here, the derivatives w.r.t. $x$ are acting on step-functions. If we parameterize the boundary by $y=\gamma(x)$, then taking the first and second derivative, respectively, yields terms including $\delta(y-\gamma(x))$ and $\delta(y-\gamma(x)) /(y-\gamma(x))$, respectively. Under certain circumstances, these may give significant contributions in the evaluation of $\langle\cdot, \cdot\rangle_{\mu_{x}}$, and we can compute explicit expressions for them.

However, the step-functions result from the approximation (5.7) of the eigenfunction $u_{1}$ in $L^{2}\left(\mu_{x}\right)$, and the scalar products include the weight $\mu_{x}$. This has mainly two consequences: (A) The eigenfunction $u_{1}$ actually is much smoother than the stepfunctions, and (B) the boundary typically is near the saddle point of the potential; i.e., the weight $\mu_{x}$ is exponentially small near the boundary. Together, these aspects render the boundary terms to be negligible in all cases considered so far by the authors. Nevertheless, one might be able to make up scenarios where these contributions cannot be neglected. However, then it is the conjecture of the authors that one should reformulate conditional averaging in terms of a set of generalized coordinates that are adapted to the boundary.

Appendix B. Projection onto dominant subspace: Details. As in section 5.2 we start with the ansatz

$$
\rho^{\epsilon}(x, y, t)=\sum_{k=0}^{M-1} c_{k}(x, t) u_{k}(x, y)
$$

Now, we first insert this into the Fokker-Planck equation (2.1) and then let the $M$ projections $\left\langle u_{k}, \cdot\right\rangle_{\mu_{x}}$ act on the resulting equation. Since the projections are all orthogonal this procedure yields $M$ independent evolution equations for the coefficients $c_{k}$ in our ansatz:

$$
\begin{aligned}
& \partial_{t} c_{0}=\left\langle\mathbf{1}, \mathcal{L}_{y} \rho^{\epsilon}\right\rangle_{\mu_{x}}, \\
& \partial_{t} c_{k}=\left\langle u_{k}, \mathcal{L}_{y} \rho^{\epsilon}\right\rangle_{\mu_{x}}+\frac{1}{\epsilon} \lambda_{k}(x) c_{k}, \quad k=1, \ldots, M-1 .
\end{aligned}
$$

The evaluation of the RHS of these equations proceeds by inserting $\rho^{\epsilon}$ :

$$
\begin{aligned}
\left\langle\mathbf{1}, \mathcal{L}_{y} \rho^{\epsilon}\right\rangle_{\mu_{x}}=\sum_{k=0}^{M-1} & \left(\left\langle\mathbf{1}, u_{k} \mathcal{L}_{y} c_{k}\right\rangle_{\mu_{x}}+c_{k}\left\langle\mathbf{1}, \mathcal{L}_{y} u_{k}\right\rangle_{\mu_{x}}\right. \\
& \left.+\sigma^{2}\left\langle\mathbf{1}, D_{x} u_{k}\right\rangle_{\mu_{x}} \cdot D_{x} c_{k}\right) .
\end{aligned}
$$

The different terms of this equation yield

$$
\begin{aligned}
\left\langle\mathbf{1}, u_{0} \mathcal{L}_{y} c_{0}\right\rangle_{\mu_{x}} & =\overline{\mathcal{L}} c_{0}, \\
\left\langle\mathbf{1}, u_{k} \mathcal{L}_{y} c_{k}\right\rangle_{\mu_{x}} & =-\left\langle u_{k}, D_{x} V\right\rangle_{\mu_{x}} \cdot D_{x} c_{k}, \quad k>0, \\
\left\langle\mathbf{1}, \mathcal{L}_{y} u_{0}\right\rangle_{\mu_{x}} & =0 \\
\left\langle\mathbf{1}, \mathcal{L}_{y} u_{k}\right\rangle_{\mu_{x}} & =\left\langle\mathbf{1}, \mathcal{L}_{y} u_{k}\right\rangle_{\mu_{x}}, \quad k>0 \\
\left\langle\mathbf{1}, D_{x} u_{0}\right\rangle_{\mu_{x}} & =0
\end{aligned}
$$


Conclusively, we get for the main coefficient

$$
\begin{gathered}
\partial_{t} c_{0}=\overline{\mathcal{L}} c_{0}+\sum_{k=1}^{M-1}\left(\left\langle\mathbf{1}, \mathcal{L}_{y} u_{k}\right\rangle_{\mu_{x}} c_{k}-\left\langle u_{k}, D_{x} V\right\rangle_{\mu_{x}} \cdot D_{x} c_{k}\right. \\
\left.+\sigma^{2}\left\langle\mathbf{1}, D_{x} u_{k}\right\rangle_{\mu_{x}} \cdot D_{x} c_{k}\right),
\end{gathered}
$$

while the other coefficients $(k>0)$ satisfy

$$
\begin{array}{r}
\partial_{t} c_{k}=\tilde{\mathcal{L}}_{k} c_{k}+\sum_{\substack{l=0 \\
l \neq k}}^{M-1}\left(\left\langle u_{k}, \mathcal{L}_{y} u_{l}\right\rangle_{\mu_{x}} c_{l}-\left\langle u_{k}, u_{l} D_{x} V\right\rangle_{\mu_{x}} \cdot D_{x} c_{l}\right. \\
\left.\quad+\sigma^{2}\left\langle u_{k}, D_{x} u_{l}\right\rangle_{\mu_{x}} \cdot D_{x} c_{l}\right)+\frac{1}{\epsilon} \lambda_{k} c_{k},
\end{array}
$$

with

$$
\tilde{\mathcal{L}}_{k}=\left\langle u_{k}, u_{k} \mathcal{L}_{y}\right\rangle_{\mu_{x}}
$$

\section{REFERENCES}

[1] M. P. Allen And D. J. Tildesley, Computer Simulations of Liquids, Clarendon, Oxford, 1987.

[2] V. I. Arnold, V. V. Kozlov, and A. I. Neishtadt, Mathematical Aspects of Classical and Celestial Mechanics, Springer, Berlin, 1993.

[3] E. Barth, K. Kuczera, B. Leimkuhler, and R. D. Skeel, Algorithms for constrained molecular dynamics, J. Comp. Chem., 16 (1995), pp. 1192-1209.

[4] P. G. Bolhuis, D. Chandler, C. Dellago, and P. Geissler, Transition path sampling: Throwing ropes over mountain passes, in the dark, Ann. Rev. Phys. Chem., 59 (2002), pp. 291-318.

[5] M. Born And V. Fock, Beweis des Adiabatensatzes, Z. Phys., 51 (1928), pp. 165-180.

[6] F. A. Bornemann, Homogenization in Time of Singularly Perturbed Mechanical Systems, Lecture Notes in Math. 1687, Springer-Verlag, 1998.

[7] F. A. Bornemann And C. Schütte, Homogenization of Hamiltonian systems with a strong constraining potential, Phys. D, 102 (1997), pp. 57-77.

[8] F. A. Bornemann And C. Schütte, On the singular limit of the quantum-classical molecular dynamics model, SIAM J. Appl. Math., 59 (1999), pp. 1208-1224.

[9] A. Bovier, V. Gayrard, and M. Klein, Metastability in Reversible Diffusion Processes II: Precise Asymptotics for Small Eigenvalues, WIAS Preprint 768, Weierstrass Institute for Applied Analysis and Stochastics, Berlin, Germany, 2002.

[10] E. A. Carter, G. Ciccotti, J. T. Hynes, and R. Kapral, Constrained reaction coordinate dynamics for the simulation of rare events, Chem. Phys. Lett., 156 (1989), pp. 472-477.

[11] A. Chorin, A. Kast, and R. Kupferman, Optimal prediction of unresolved dynamics, Proc. Natl. Acad. Sci. USA, 95 (1998), pp. 4094-4098.

[12] A. J. Chorin, O. H. HAld, AND R. Kupferman, Optimal prediction and the Mori-Zwanzig representation of irreversible processes, Proc. Natl. Acad. Sci. USA, 97 (2000), pp. 2968-2973.

[13] E. B. Davies, Metastable states of symmetric Markov semigroups II, J. London Math. Soc. (2), 26 (1982), pp. 541-556.

[14] M. Dellnitz And O. Junge, On the approximation of complicated dynamical behavior, SIAM J. Numer. Anal., 36 (1999), pp. 491-515.

[15] P. Deuflhard, J. Hermans, B. Leimkuhler, A. E. Mark, S. Reich, and R. D. Skeel, eds., Computational Molecular Dynamics: Challenges, Methods, Ideas, Lect. Notes Comput. Sci. Eng. 4, Springer-Verlag, Berlin, 1999.

[16] P. Deuflhard, W. Huisinga, A. Fischer, And C. Schütte, Identification of almost invariant aggregates in reversible nearly uncoupled Markov chains, Linear Algebra Appl., 315 (2000), pp. $39-59$.

[17] W. E, W. Ren, and E. Vanden-Eijnden, Probing multi-scale energy landscapes using the string method, Phys. Rev. Lett., submitted. 
[18] C. Fermanian-Kammerer and C. Lasser, Wigner measures and codimension two crossings, J. Math. Phys., 44 (2003), pp. 507-527.

[19] A. Fischer, An Uncoupling-Coupling Method for Markov Chain Monte Carlo Simulations with an Application to Biomolecules, Ph.D. thesis, Free University Berlin, Berlin, Germany, 2003.

[20] A. Fischer, C. Schütte, P. Deuflhard, And F. Cordes, Hierarchical uncoupling-coupling of metastable conformations, in Computational Methods for Macromolecules: Challenges and Applications, Lect. Notes Comput. Sci. Eng. 24, T. Schlick and H. H. Gan, eds., Springer, Heidelberg, 2002.

[21] M. Fixman, Classical statistical mechanics of constraints: A theorem and application to polymers, Proc. Nat. Acad. Sci. U.S.A., 71 (1974), pp. 3050-3053.

[22] M. Freiduin, The averaging principle and theorems on large deviations, Russian Math. Surveys, 33 (1978), pp. 107-160.

[23] M. Freidlin and A. Wentzell, Random Perturbations of Dynamical Systems, Grundlehren Math. Wiss. 260, Springer, New York, 1984.

[24] A. Gelman and X.-L. Meng, Simulating normalizing constants: From importance sampling to bridge sampling to path sampling, Statist. Sci., 13 (1998), pp. 163-185.

[25] C. J. GEYER, Estimating Normalizing Constants and Reweighting Mixtures in Markov Chain Monte Carlo, Technical report 568, University of Minnesota, Minneapolis, MN, 1994.

[26] G. Hagedorn, A time dependent Born-Oppenheimer approximation, Comm. Math. Phys., 77 (1980), pp. 1-19.

[27] G. A. Hagedorn, Molecular propagation through electron energy level crossings, Mem. Amer. Math. Soc., 111 (536) (1994), pp. 1-130.

[28] I. Horenko, B. Schmidt, And C. Schütte, A theoretical model for molecules interacting with intense laser pulses: The Floquet-based quantum-classical Liouville equation, J. Chem. Phys., 115 (2001), pp. 5733-5743.

[29] W. Huisinga, Metastability of Markovian Systems: A Transfer Operator Based Approach in Application to Molecular Dynamics, Ph.D. thesis, Free University Berlin, Berlin, Germany, 2001.

[30] W. Huisinga, S. Meyn, and C. Schütte, Phase transitions and metastability in Markovian and molecular systems, Ann. Appl. Probab., 14 (2004), pp. 419-458.

[31] Y. KIfER, Averaging in dynamical systems and large deviations, Invent. Math., 110 (1992), pp. 337-370.

[32] Y. KIFER, Stochastic versions of Anosov's and Neistadt's theorems on averaging, Stoch. Dyn., 1 (2001), pp. 1-21.

[33] Y. I. KIFER, $L^{2}$ diffusion approximation for slow motion in averaging, Stoch. Dyn., 3 (2003), pp. 213-246.

[34] K. KuCZera, One- and multidimensional conformational free energy simulations, J. Comp. Chem., 17 (1996), pp. 1726-1749.

[35] K. Kuczera, Exploration of peptide free energy surfaces, in Computational Molecular Dynamics: Challenges, Methods, Ideas, Lect. Notes Comput. Sci. Eng. 4, Springer-Verlag, Berlin, 1999, pp. 161-175.

[36] R. Kupferman and A. Stuart, Fitting SDE Models to Nonlinear Kac-Zwanzig Heat Bath Models, Warwick Preprint 31/2002, University of Warwick, Coventry, UK, 2002.

[37] T. G. KuRTz, A limit theorem for perturbed operator semigroups with applications to random evolutions, J. Funct. Anal., 12 (1973), pp. 55-67.

[38] L. D. Landau, Zur Theorie der Energieübertragung bei Stößen, Phys. Z. Sowjetunion, 2 (1932), p. 46.

[39] A. J. Majda, I. Timofeyev, and E. Vanden-Eijnden, A mathematical framework for stochastic climate models, Comm. Pure Appl. Math., 54 (2001), pp. 891-974.

[40] A. J. Majda, I. Timofeyev, and E. Vanden-Eijnden, A priori tests of a stochastic mode reduction strategy, Phys. D, 170 (2002), pp. 206-252.

[41] J. Mattingly, A. M. Stuart, and D. J. Higham, Ergodicity for SDEs and approximations: Locally Lipschitz vector fields and degenerated noise, Stochastic Process. Appl., 10 (2002), pp. 185-232.

[42] S. Meyn And R. Tweedie, Markov Chains and Stochastic Stability, Springer, Berlin, 1993.

[43] H. MorI, Transport collective motion and Brownian motion, Prog. Theoret. Phys. Suppl., 33 (1965), pp. 423-455.

[44] I. PaVlyukevich, Stochastic Resonance, Logos, Berlin, 2002.

[45] S. REICH, Smoothed Langevin dynamics of highly oscillatory systems, Phys. D, 138 (2000), pp. 210-224.

[46] H. Rubin and P. Ungar, Motion under strong constraining force, Comm. Pure Appl. Math., 
10 (1957), pp. 65-87.

[47] R. Samudrala And J. Moult, An all-atom distance-dependent conditional probability discriminatory function for protein structure prediction, J. Mol. Biol., 275 (1998), pp. 895-916.

[48] J. Sanders And F. Verhulst, Averaging Methods in Nonlinear Dynamical Systems, Springer, New York, 1985.

[49] T. SChneider AND E. Stoll, Molecular dynamics study of a three-dimensional one-component model for distortive phase-transitions, Phys. Rev. B, 17 (1978), pp. 1302-1322.

[50] C. Schütte, A. Fischer, W. Huisinga, and P. Deuflhard, A direct approach to conformational dynamics based on hybrid Monte Carlo, J. Comput. Phys., 151 (1999), pp. 146-168.

[51] C. Schütte And W. Huisinga, Biomolecular conformations can be identified as metastable sets of molecular dynamics, in Handbook of Numerical Analysis, Vol. 10, P. G. Ciarlet, ed., North-Holland, Amsterdam, 2003, pp. 699-744.

[52] H. Spohn And S. Teufel, Adiabatic decoupling and time-dependent Born-Oppenheimer theory, Comm. Math. Phys., 224 (2001), pp. 113-132.

[53] M. Sprik And G. Ciccotti, Free energy from constrained molecular dynamics, J. Chem. Phys., 109 (1998), pp. 7737-7744.

[54] F. TAKEns, Motion under the influence of a strong constraining force, in Global Theory of Dynamical Systems, Lecture Notes in Math. 819, Z. Niteki and C. Robinson, eds., Springer, Evanston, 1979, pp. 425-445.

[55] C. Zener, Nonadiabatic crossing of energy levels, Proc. Roy. Soc London Ser. A, 7 (1932), p. 696.

[56] R. ZwanZIG, Nonlinear generalized Langevin equations, J. Statist. Phys., 9 (1973), pp. 215-220. 